\title{
K-GROUPS FOR RINGS OF FINITE COHEN-MACAULAY TYPE
}

\author{
HENRIK HOLM
}

\begin{abstract}
For a local Cohen-Macaulay ring $R$ of finite CM-type, Yoshino has applied methods of Auslander and Reiten to compute the Grothendieck group $\mathrm{K}_{0}$ of the category $\bmod R$ of finitely generated $R$-modules. For the same type of rings, we compute in this paper the first Quillen $\mathrm{K}$-group $\mathrm{K}_{1}(\bmod R)$. We also describe the group homomorphism $R^{*} \rightarrow \mathrm{K}_{1}(\bmod R)$ induced by the inclusion functor $\operatorname{proj} R \rightarrow \bmod R$ and illustrate our results with concrete examples.
\end{abstract}

\section{INTRODUCTION}

Throughout this introduction, $R$ denotes a commutative noetherian local CohenMacaulay ring. The lower $\mathrm{K}$-groups of $R$ are known: $\mathrm{K}_{0}(R) \cong \mathbb{Z}$ and $\mathrm{K}_{1}(R) \cong R^{*}$. For $n \in\{0,1\}$ the classical K-group $\mathrm{K}_{n}(R)$ of the ring coincides with Quillen's Kgroup $\mathrm{K}_{n}(\operatorname{proj} R$ ) of the exact category of finitely generated projective $R$-modules; and if $R$ is regular, then Quillen's resolution theorem shows that the inclusion functor $\operatorname{proj} R \rightarrow \bmod R$ induces an isomorphism $\mathrm{K}_{n}(\operatorname{proj} R) \cong \mathrm{K}_{n}(\bmod R)$. If $R$ is non-regular, then these groups are usually not isomorphic. The groups $\mathrm{K}_{n}(\bmod R)$ are often denoted $\mathrm{G}_{n}(R)$ and they are classical objects of study called the G-theory of $R$. A celebrated result of Quillen is that G-theory is well-behaved under (Laurent) polynomial extensions: $\mathrm{G}_{n}(R[t]) \cong \mathrm{G}_{n}(R)$ and $\mathrm{G}_{n}\left(R\left[t, t^{-1}\right]\right) \cong \mathrm{G}_{n}(R) \oplus \mathrm{G}_{n-1}(R)$.

Auslander and Reiten [4] and Butler [9] computed $\mathrm{K}_{0}(\bmod \Lambda)$ for an Artin algebra $\Lambda$ of finite representation type. Using similar techniques, Yoshino 32 computed $\mathrm{K}_{0}(\bmod R)$ in the case where $R$ has finite (as opposed to tame or wild) CM-type:

Theorem (Yoshino [32, thm. (13.7)]). Assume that $R$ is henselian and that it has a dualizing module. If $R$ has finite $C M$-type, then there is a group isomorphism,

$$
\mathrm{K}_{0}(\bmod R) \cong \operatorname{Coker} \Upsilon,
$$

where $\Upsilon: \mathbb{Z}^{t} \rightarrow \mathbb{Z}^{t+1}$ is the Auslander-Reiten homomorphism from (2.3).

We mention that Yoshino's result is as much a contribution to algebraic Ktheory as it is to the representation theory of the category MCM $R$ of maximal Cohen-Macaulay $R$-modules. Indeed, the inclusion functor $\operatorname{MCM} R \rightarrow \bmod R$ induces an isomorphism $\mathrm{K}_{n}(\mathrm{MCM} R) \cong \mathrm{K}_{n}(\bmod R)$ for every $n$. The theory of maximal Cohen-Macaulay modules, which originates from algebraic geometry and integral representations of finite groups, is a highly active area of research.

In this paper, we build upon results and techniques of Auslander and Reiten [4, Bass [7, Lam [20, Leuschke [21, Quillen [24, Vaserstein [28, 29], and Yoshino [32]

2010 Mathematics Subject Classification. 13C14, 13D15, 19B28.

Key words and phrases. Auslander-Reiten sequence; Bass' universal determinant group; finite Cohen-Macaulay type; maximal Cohen-Macaulay module; Quillen's K-theory. 
to compute the group $\mathrm{K}_{1}(\bmod R)$ when $R$ has finite CM-type. Our main result is Theorem (2.12); it asserts that there is an isomorphism,

$$
\mathrm{K}_{1}(\bmod R) \cong \operatorname{Aut}_{R}(M)_{\mathrm{ab}} / \Xi,
$$

where $M$ is any representation generator of the category of maximal Cohen-Macaulay $R$-modules and $\operatorname{Aut}_{R}(M)_{\mathrm{ab}}$ is the abelianization of its automorphism group. The subgroup $\Xi$ is more complicated to describe; it is determined by the AuslanderReiten sequences and defined in (2.10). Observe that in contrast to $\mathrm{K}_{0}(\bmod R)$, the group $\mathrm{K}_{1}(\bmod R)$ is usually not finitely generated.

We also prove that if one writes $M=R \oplus M^{\prime}$, then the group homomorphism $R^{*} \cong \mathrm{K}_{1}(\operatorname{proj} R) \rightarrow \mathrm{K}_{1}(\bmod R)$ induced by the inclusion functor $\operatorname{proj} R \rightarrow \bmod R$ can be identified with the map

$$
\lambda: R^{*} \longrightarrow \operatorname{Aut}_{R}(M)_{\mathrm{ab}} / \Xi \quad \text { given by } \quad r \longmapsto\left(\begin{array}{cc}
r 1_{R} & 0 \\
0 & 1_{M^{\prime}}
\end{array}\right) .
$$

The paper is organized as follows: In Section 2 we formulate our main result, Theorem (2.12). This theorem is not proved until Section 8 and the intermediate Sections 3 (on the Gersten-Sherman transformation), 4 (on Auslander's and Reiten's theory for coherent pairs), 5 (on Vaserstein's result for semilocal rings), 6 (on certain equivalences of categories), and 7 (on Yoshino's results for the abelian category $\mathcal{Y}$ ) prepare the ground.

In Sections 9 and 10 we apply our main theorem to compute the group $\mathrm{K}_{1}(\bmod R)$ and the homomorphism $\lambda: R^{*} \rightarrow \mathrm{K}_{1}(\bmod R)$ in some concrete examples. E.g. for the simple curve singularity $R=k \llbracket T^{2}, T^{3} \rrbracket$ we obtain $\mathrm{K}_{1}(\bmod R) \cong k \llbracket T \rrbracket^{*}$ and show that the homomorphism $\lambda: k \llbracket T^{2}, T^{3} \rrbracket^{*} \rightarrow k \llbracket T \rrbracket^{*}$ is the inclusion. It is well-known that if $R$ is artinian with residue field $k$, then one has $\mathrm{K}_{1}(\bmod R) \cong k^{*}$. We apply Theorem (2.12) to confirm this isomorphism for the ring $R=k[X] /\left(X^{2}\right)$ of dual numbers and to show that the homomorphism $\lambda: R^{*} \rightarrow k^{*}$ is given by $a+b X \mapsto a^{2}$.

We end this introduction by mentioning a related preprint 23 of Navkal. Although the present work and the paper of Navkal have been written completely independently (this fact is also pointet out in the latest version of 23]), there is a significant overlap between the two manuscripts: Navkal's main result [23, thm. 1.2] is the existence of a long exact sequence involving the G-theory of the rings $R$ and $\operatorname{End}_{R}(M)^{\mathrm{op}}$ (where $M$ is a particular representation generator of the category of maximal Cohen-Macaulay $R$-modules) and the K-theory of certain division rings. In Section 5 in loc. cit., Navkal applies his main result to give some description of the group $\mathrm{K}_{1}(\bmod R)$ for the ring $R=k \llbracket T^{2}, T^{2 n+1} \rrbracket$ where $n \geqslant 1$. We point out that the techniques used in this paper and in Navkal's work are quite different.

\section{Formulation of the Main Theorem}

Let $R$ be a commutative noetherian local Cohen-Macaulay ring. By $\bmod R$ we denote the abelian category of finitely generated $R$-modules. The exact categories of finitely generated projective modules and of maximal Cohen-Macaulay modules over $R$ are written $\operatorname{proj} R$ and $\mathrm{MCM} R$, respectively. The goal of this section is to state our main Theorem (2.12); its proof is postponed to Section 8 .

(2.1) Setup. Throughout this paper, $(R, \mathfrak{m}, k)$ is a commutative noetherian local Cohen-Macaulay ring satisfying the following assumptions.

(1) $R$ is henselian. 
(2) $R$ admits a dualizing module.

(3) $R$ has finite $C M$-type, that is, up to isomorphism, there are only finitely many non-isomorphic indecomposable maximal Cohen-Macaulay $R$-modules.

Note that (1) and (2) hold if $R$ is $\mathfrak{m}$-adically complete. Since $R$ is henselian, the category $\bmod R$ is Krull-Schmidt by [32, prop. (1.18)]; this fact will be important a number of times in this paper.

Set $M_{0}=R$ and let $M_{1}, \ldots, M_{t}$ be a set of representatives for the isomorphism classes of non-free indecomposable maximal Cohen-Macaulay $R$-modules. Let $M$ be any representation generator of $\mathrm{MCM} R$, that is, a finitely generated $R$-module such that $\operatorname{add}_{R} M=\operatorname{MCM} R$ (where $\operatorname{add}_{R} M$ denotes the category of $R$-modules that are isomorphic to a direct summand of some finite direct sum of copies of $M$ ). For example, $M$ could be the square-free module

$$
M=M_{0} \oplus M_{1} \oplus \cdots \oplus M_{t} .
$$

We denote by $E=\operatorname{End}_{R}(M)$ the endomorphism ring of $M$.

It follows from [32, thm. (4.22)] that $R$ is an isolated singularity, and hence by loc. cit. thm. (3.2) the category MCM $R$ admits Auslander-Reiten sequences. Let

$$
0 \longrightarrow \tau\left(M_{j}\right) \longrightarrow X_{j} \longrightarrow M_{j} \longrightarrow 0 \quad(1 \leqslant j \leqslant t)
$$

be the Auslander-Reiten sequence in MCM $R$ ending in $M_{j}$, where $\tau$ is the Auslander-Reiten translation.

(2.2) Remark. The one-dimensional Cohen-Macaulay rings of finite CM-type are classified by Cimen [10, 11, Drozd and Roüter [12, Green and Reiner [18, and Wiegand [30, 31]. The two-dimensional complete Cohen-Macaulay rings of finite CM-type that contains the complex numbers are classified by Auslander [2, Esnault 14, and Herzog $\llbracket 19$. They are the invariant rings $R=\mathbb{C} \llbracket X, Y \rrbracket^{G}$ where $G$ is a nontrivial finite subgroup of $\mathrm{GL}_{2}(\mathbb{C})$. In this case, $M=\mathbb{C} \llbracket X, Y \rrbracket$ is a representation generator for MCM $R$ which, unlike the one in (2.1.1), need not be square-free.

(2.3) Definition. For each Auslander-Reiten sequence (2.1.2) we have

$$
X_{j} \cong M_{0}^{n_{0 j}} \oplus M_{1}^{n_{1 j}} \oplus \cdots \oplus M_{t}^{n_{t j}}
$$

for uniquely determined $n_{0 j}, n_{1 j}, \ldots, n_{t j} \geqslant 0$. Consider the element,

$$
\tau\left(M_{j}\right)+M_{j}-n_{0 j} M_{0}-n_{1 j} M_{1}-\cdots-n_{t j} M_{t},
$$

in the free abelian group $\mathbb{Z} M_{0} \oplus \mathbb{Z} M_{1} \oplus \cdots \oplus \mathbb{Z} M_{t}$, and write this element as,

$$
y_{0 j} M_{0}+y_{1 j} M_{1}+\cdots+y_{t j} M_{t},
$$

where $y_{0 j}, y_{1 j}, \ldots, y_{t j} \in \mathbb{Z}$. Define the Auslander-Reiten matrix $\Upsilon$ as the $(t+1) \times t$ matrix with entries in $\mathbb{Z}$ whose $j^{\prime}$ th column is $\left(y_{0 j}, y_{1 j}, \ldots, y_{t j}\right)$. When $\Upsilon$ is viewed as a homomorphism of abelian groups $\Upsilon: \mathbb{Z}^{t} \rightarrow \mathbb{Z}^{t+1}$ (elements in $\mathbb{Z}^{t}$ and $\mathbb{Z}^{t+1}$ are viewed as column vectors), we refer to it as the Auslander-Reiten homomorphism.

(2.4) Example. Let $R=\mathbb{C} \llbracket X, Y, Z \rrbracket /\left(X^{3}+Y^{4}+Z^{2}\right)$. Besides $M_{0}=R$ there are exactly $t=6$ non-isomorphic indecomposable maximal Cohen-Macaulay modules, 
and the Auslander-Reiten sequences have the following form,

$$
\begin{array}{r}
0 \longrightarrow M_{1} \longrightarrow M_{2} \longrightarrow M_{1} \longrightarrow 0 \\
0 \longrightarrow M_{2} \longrightarrow M_{1} \oplus M_{3} \longrightarrow M_{2} \longrightarrow 0 \\
0 \longrightarrow M_{3} \longrightarrow M_{2} \oplus M_{4} \oplus M_{6} \longrightarrow M_{3} \longrightarrow 0 \\
0 \longrightarrow M_{4} \longrightarrow M_{3} \oplus M_{5} \longrightarrow M_{4} \longrightarrow 0 \\
0 \longrightarrow M_{5} \longrightarrow M_{4} \longrightarrow M_{5} \longrightarrow 0 \\
0 \longrightarrow M_{6} \longrightarrow M_{0} \oplus M_{3} \longrightarrow M_{6} \longrightarrow 0 ;
\end{array}
$$

see [32, (13.9)]. The $7 \times 6$ Auslander-Reiten matrix $\Upsilon$ is therefore given by

$$
\Upsilon=\left(\begin{array}{cccccc}
0 & 0 & 0 & 0 & 0 & -1 \\
2 & -1 & 0 & 0 & 0 & 0 \\
-1 & 2 & -1 & 0 & 0 & 0 \\
0 & -1 & 2 & -1 & 0 & -1 \\
0 & 0 & -1 & 2 & -1 & 0 \\
0 & 0 & 0 & -1 & 2 & 0 \\
0 & 0 & -1 & 0 & 0 & 2
\end{array}\right)
$$

In this case, the Auslander-Reiten homomorphism $\Upsilon: \mathbb{Z}^{6} \rightarrow \mathbb{Z}^{7}$ is clearly injective.

One hypothesis in our main result, Theorem (2.12) below, is that the AuslanderReiten homomorpism $\Upsilon$ over the ring $R$ in question is injective. We are not aware of an example where $\Upsilon$ is not injective. The following lemma covers the situation of the rational double points, that is, the invariant rings $R=k \llbracket X, Y \rrbracket^{G}$, where $k$ is an algebraically closed field of characteristic 0 and $G$ is a non-trivial finite subgroup of $\mathrm{SL}_{2}(k)$; see [5].

(2.5) Lemma. Assume that $R$ is complete, integrally closed, non-regular, Gorenstein, of Krull dimension 2, and that the residue field $k$ is algebraically closed. Then the Auslander-Reiten homomorphism $\Upsilon$ is injective.

Proof. Let $1 \leqslant j \leqslant t$ be given and consider the expression

$$
\tau\left(M_{j}\right)+M_{j}-n_{0 j} M_{0}-n_{1 j} M_{1}-\cdots-n_{t j} M_{t}=y_{0 j} M_{0}+y_{1 j} M_{1}+\cdots+y_{t j} M_{t}
$$

in the free abelian group $\mathbb{Z} M_{0} \oplus \mathbb{Z} M_{1} \oplus \cdots \oplus \mathbb{Z} M_{t}$, see Definition (2.3). Let $\Gamma$ be the Auslander-Reiten quiver of MCM R. We recall from [5, thm. 1] that the arrows in $\Gamma$ occur in pairs $\circ \rightleftarrows \circ$, and that collapsing each pair to an undirected edge gives an extended Dynkin diagram $\tilde{\Delta}$. Moreover, removing the vertex corresponding to $M_{0}=R$ and any incident edges gives a Dynkin graph $\Delta$.

Now, $X_{j}$ has a direct summand $M_{k}$ if and only if there is an arrow $M_{k} \rightarrow M_{j}$ in $\Gamma$. Also, the Auslander-Reiten translation $\tau$ satisfies $\tau\left(M_{j}\right)=M_{j}$ by [5, proof of thm. 1]. Combined with the structure of the Auslander-Reiten quiver, this means that

$$
y_{k j}=\left\{\begin{array}{cl}
2 & \text { if } k=j, \\
-1 & \text { if there is an edge } M_{k}-M_{j} \text { in } \tilde{\Delta}, \\
0 & \text { otherwise } .
\end{array}\right.
$$

Hence the $t \times t$ matrix $\Upsilon_{0}$ with $\left(y_{1 j}, \ldots, y_{t j}\right)$ as $j$ 'th column, where $1 \leqslant j \leqslant t$, is the Cartan matrix of the Dynkin graph $\Delta$; cf. [8, def. 4.5.3]. This matrix is invertible by [15, exer. (21.18)]. Deleting the first row $\left(y_{01}, \ldots, y_{0 t}\right)$ in the Auslander-Reiten matrix $\Upsilon$, we get the invertible matrix $\Upsilon_{0}$, and consequently, $\Upsilon: \mathbb{Z}^{t} \rightarrow \mathbb{Z}^{t+1}$ determines an injective homomorphism. 
For a group $G$ we denote by $G_{\mathrm{ab}}$ its abelianization, i.e. $G_{\mathrm{ab}}=G /[G, G]$, where $[G, G]$ is the commutator subgroup of $G$.

We refer to the following as the tilde construction. It associates to every automorphism $\alpha: X \rightarrow X$ of a maximal Cohen-Macaulay module $X$ an automorphism $\tilde{\alpha}: M^{q} \rightarrow M^{q}$ of the smallest power $q$ of the representation generator $M$ such that $X$ is a direct summand of $M^{q}$.

(2.6) Construction. The chosen representation generator $M$ for $\mathrm{MCM} R$ has the form $M=M_{0}^{m_{0}} \oplus \cdots \oplus M_{t}^{m_{t}}$ for uniquely determined integers $m_{0}, \ldots, m_{t}>0$. For any module $X=M_{0}^{n_{0}} \oplus \cdots \oplus M_{t}^{n_{t}}$ in MCM $R$, we define natural numbers,

$$
\begin{aligned}
q & =q(X)=\min \left\{p \in \mathbb{N} \mid p m_{j} \geqslant n_{j} \text { for all } 0 \leqslant j \leqslant t\right\}, \text { and } \\
v_{j} & =v_{j}(X)=q m_{j}-n_{j} \geqslant 0,
\end{aligned}
$$

and a module $Y=M_{0}^{v_{0}} \oplus \cdots \oplus M_{t}^{v_{t}}$ in MCM $R$. Let $\psi: X \oplus Y \stackrel{\cong}{\longrightarrow} M^{q}$ be the $R$ isomorphism that maps an element

$$
\left(\left(\underline{x}_{0}, \ldots, \underline{x}_{t}\right),\left(\underline{y}_{0}, \ldots, \underline{y}_{t}\right)\right) \in X \oplus Y=\left(M_{0}^{n_{0}} \oplus \cdots \oplus M_{t}^{n_{t}}\right) \oplus\left(M_{0}^{v_{0}} \oplus \cdots \oplus M_{t}^{v_{t}}\right),
$$

where $\underline{x}_{j} \in M_{j}^{n_{j}}$ and $\underline{y}_{j} \in M_{j}^{v_{j}}$, to the element

$$
\left(\left(\underline{z}_{01}, \ldots, \underline{z}_{t 1}\right), \ldots,\left(\underline{z}_{0 q}, \ldots, \underline{z}_{t q}\right)\right) \in M^{q}=\left(M_{0}^{m_{0}} \oplus \cdots \oplus M_{t}^{m_{t}}\right)^{q},
$$

where $\underline{z}_{j 1}, \ldots, \underline{z}_{j q} \in M_{j}^{m_{j}}$ are given by $\left(\underline{z}_{j 1}, \ldots, \underline{z}_{j q}\right)=\left(\underline{x}_{j}, \underline{y}_{j}\right) \in M_{j}^{q m_{j}}=M_{j}^{n_{j}+v_{j}}$.

Now, given $\alpha$ in $\operatorname{Aut}_{R}(X)$, we define $\tilde{\alpha}$ to be the uniquely determined element in $\operatorname{Aut}_{R}\left(M^{q}\right)$ that makes the following diagram commutative,

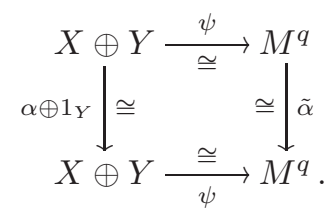

The automorphism $\tilde{\alpha}$ of $M^{q}$ has the form $\tilde{\alpha}=\left(\tilde{\alpha}_{i j}\right)$ for uniquely determined endomorphisms $\tilde{\alpha}_{i j}$ of $M$, that is, $\tilde{\alpha}_{i j} \in E=\operatorname{End}_{R}(M)$. Hence $\tilde{\alpha}=\left(\tilde{\alpha}_{i j}\right)$ can naturally be viewed as an invertible $q \times q$ matrix with entries in $E$.

$$
\begin{gathered}
\text { Example. Let } M=M_{0} \oplus \cdots \oplus M_{t} \text { and } X=M_{j} \text {. Then } q=1 \text { and } \\
\qquad Y=M_{0} \oplus \cdots \oplus M_{j-1} \oplus M_{j+1} \oplus \cdots \oplus M_{t} .
\end{gathered}
$$

The isomorphism $\psi: X \oplus Y \rightarrow M$ maps $\left(x_{j},\left(x_{0}, \ldots, x_{j-1}, x_{j+1}, \ldots, x_{t}\right)\right)$ in $X \oplus Y$ to $\left(x_{0}, \ldots, x_{j-1}, x_{j}, x_{j+1}, \ldots, x_{t}\right)$ in $M$. Therefore, for $\alpha \in \operatorname{Aut}_{R}(X)=\operatorname{Aut}_{R}\left(M_{j}\right)$, Construction (2.6) yields the following automorphism of $M$,

$$
\tilde{\alpha}=\psi\left(\alpha \oplus 1_{Y}\right) \psi^{-1}=1_{M_{0}} \oplus \cdots \oplus 1_{M_{j-1}} \oplus \alpha \oplus 1_{M_{j+1}} \oplus \cdots \oplus 1_{M_{t}},
$$

which is an invertible $1 \times 1$ matrix with entry in $E=\operatorname{End}_{R}(M)$.

The following result on Auslander-Reiten sequences is quite standard. We provide a few proof details along with the appropriate references.

(2.8) Proposition. Let there be given Auslander-Reiten sequences in MCM $R$,

$$
0 \longrightarrow \tau(M) \longrightarrow X \longrightarrow M \longrightarrow 0 \text { and } 0 \longrightarrow \tau\left(M^{\prime}\right) \longrightarrow X^{\prime} \longrightarrow M^{\prime} \longrightarrow 0 \text {. }
$$


If $\alpha: M \rightarrow M^{\prime}$ is a homomorphism, then there exist homomorphisms $\beta$ and $\gamma$ that make the following diagram commutative,

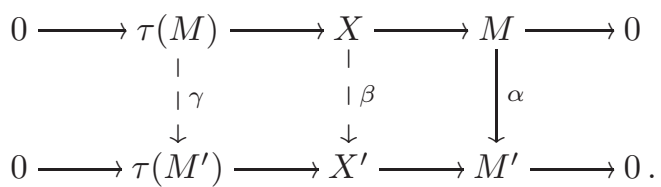

Furthermore, if $\alpha$ is an isomorphism, then so are $\beta$ and $\gamma$.

Proof. Write $\rho: X \rightarrow M$ and $\rho^{\prime}: X^{\prime} \rightarrow M^{\prime}$. It suffices to prove the existence of $\beta$ such that $\rho^{\prime} \beta=\alpha \rho$, because then the existence of $\gamma$ follows from diagram chasing.

As $0 \rightarrow \tau\left(M^{\prime}\right) \rightarrow X^{\prime} \rightarrow M^{\prime} \rightarrow 0$ is an Auslander-Reiten sequence, it suffices by [32, lem. (2.9)] to show that $\alpha \rho: X \rightarrow M^{\prime}$ is not a split epimorphism. Suppose that there do exist $\tau: M^{\prime} \rightarrow X$ with $\alpha \rho \tau=1_{M^{\prime}}$. Hence $\alpha$ is a split epimorphism. As $M$ is indecomposable, $\alpha$ must be an isomorphism. Thus $\rho \tau \alpha=\alpha^{-1}(\alpha \rho \tau) \alpha=1_{M}$, which contradicts the fact that $\rho$ is not a split epimorphism.

The fact that $\beta$ and $\gamma$ are isomorphisms if $\alpha$ is so follows from [32, lem. (2.4)].

The choice requested in the following construction is possible by Proposition (2.8).

(2.9) Construction. Choose for each $1 \leqslant j \leqslant t$ and every $\alpha \in \operatorname{Aut}_{R}\left(M_{j}\right)$ elements $\beta_{j, \alpha} \in \operatorname{Aut}_{R}\left(X_{j}\right)$ and $\gamma_{j, \alpha} \in \operatorname{Aut}_{R}\left(\tau\left(M_{j}\right)\right)$ that make the next diagram commute,

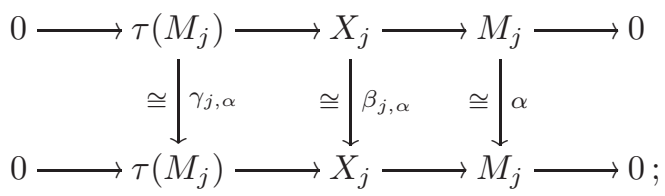

here the row(s) is the $j^{\prime}$ th Auslander-Reiten sequence (2.1.2).

As shown in Lemma (5.1), the endomorphism ring $E=\operatorname{End}_{R}(M)$ of the chosen representation generator $M$ is semilocal, that is, $E / \mathrm{J}(E)$ is semisimple. Thus, if the ground ring $R$, and hence also the endomorphism ring $E$, is an algebra over the residue field $k$ and $\operatorname{char}(k) \neq 2$, then a result by Vaserstein [29, thm. 2] yields that the canonical homomorphism $\theta_{E}: E_{\mathrm{ab}}^{*} \rightarrow \mathrm{K}_{1}^{\mathrm{C}}(E)$ is an isomorphism. Here $\mathrm{K}_{1}^{\mathrm{C}}(E)$ is the classical $\mathrm{K}_{1}$-group of the ring $E$; see (3.1). Its inverse,

$$
\theta_{E}^{-1}=\operatorname{det}_{E}: \mathrm{K}_{1}^{\mathrm{C}}(E) \longrightarrow E_{\mathrm{ab}}^{*}=\operatorname{Aut}_{R}(M)_{\mathrm{ab}},
$$

is called the generalized determinant map. The details are discussed in Section 5 . We are now in a position to define the subgroup $\Xi$ of $\operatorname{Aut}_{R}(M)_{\text {ab }}$ that appears in our main Theorem (2.12) below.

(2.10) Definition. Let $(R, \mathfrak{m}, k)$ be a ring satisfying the hypotheses in Setup (2.1). Assume, in addition, that $R$ is an algebra over $k$ and that one has $\operatorname{char}(k) \neq 2$. Define a subgroup $\Xi$ of $\operatorname{Aut}_{R}(M)_{\text {ab }}$ as follows.

- Choose for each $1 \leqslant j \leqslant t$ and each $\alpha \in \operatorname{Aut}_{R}\left(M_{j}\right)$ elements $\beta_{j, \alpha} \in \operatorname{Aut}_{R}\left(X_{j}\right)$ and $\gamma_{j, \alpha} \in \operatorname{Aut}_{R}\left(\tau\left(M_{j}\right)\right)$ as in Construction (2.9).

- Let $\tilde{\alpha}, \tilde{\beta}_{j, \alpha}$, and $\tilde{\gamma}_{j, \alpha}$ be the invertible matrices with entries in $E$ obtained by applying the tilde construction (2.6) to $\alpha, \beta_{j, \alpha}$, and $\gamma_{j, \alpha}$. 
Let $\Xi$ be the subgroup of $\operatorname{Aut}_{R}(M)_{\text {ab }}$ generated by the elements

$$
\left(\operatorname{det}_{E} \tilde{\alpha}\right)\left(\operatorname{det}_{E} \tilde{\beta}_{j, \alpha}\right)^{-1}\left(\operatorname{det}_{E} \tilde{\gamma}_{j, \alpha}\right),
$$

where $j$ ranges over $\{1, \ldots, t\}$ and $\alpha$ over $\operatorname{Aut}_{R}\left(M_{j}\right)$.

A priori the definition of the group $\Xi$ involves certain choices. However, it follows from Proposition (8.8) that $\Xi$ is actually independent of the choices made.

(2.11) Remark. In specific examples it is convenient to consider the simplest possible representation generator $M=M_{0} \oplus M_{1} \oplus \cdots \oplus M_{t}$. In this case, Example (2.7) shows that $\tilde{\alpha}$ and $\tilde{\gamma}_{j, \alpha}$ are $1 \times 1$ matrices with entries in $E$, that is, $\tilde{\alpha}, \tilde{\gamma}_{j, \alpha} \in E^{*}$, and consequently $\operatorname{det}_{E} \tilde{\alpha}=\tilde{\alpha}$ and $\operatorname{det}_{E} \tilde{\gamma}_{j, \alpha}=\tilde{\gamma}_{j, \alpha}$ as elements in $E_{\mathrm{ab}}^{*}$.

We are now in a position to state our main result.

(2.12) Theorem. Let $(R, \mathfrak{m}, k)$ be a ring satisfying the hypotheses in Setup (2.1). Assume that $R$ is an algebra over its residue field $k$ with $\operatorname{char}(k) \neq 2$, and that the Auslander-Reiten homomorphism $\Upsilon: \mathbb{Z}^{t} \rightarrow \mathbb{Z}^{t+1}$ from Definition (2.3) is injective.

Let $M$ be any representation generator of MCM $R$. There is an isomorphism,

$$
\mathrm{K}_{1}(\bmod R) \cong \operatorname{Aut}_{R}(M)_{\mathrm{ab}} / \Xi
$$

where $\Xi$ is the subgroup of $\operatorname{Aut}_{R}(M)_{\mathrm{ab}}$ given in Definition (2.10).

Furthermore, if inc: $\operatorname{proj} R \rightarrow \bmod R$ is the inclusion functor and $M=R \oplus M^{\prime}$, then $\mathrm{K}_{1}(\mathrm{inc}): \mathrm{K}_{1}(\operatorname{proj} R) \rightarrow \mathrm{K}_{1}(\bmod R)$ may be identified with the homomorphism,

$$
\lambda: R^{*} \longrightarrow \operatorname{Aut}_{R}(M)_{\mathrm{ab}} / \Xi \quad \text { given by } \quad r \longmapsto\left(\begin{array}{cc}
r 1_{R} & 0 \\
0 & 1_{M^{\prime}}
\end{array}\right) .
$$

As mentioned in the introduction, the proof of Theorem (2.12) spans Sections 3 to 8 . Applications and examples are presented in Sections 9 and 10. The interested reader could go ahead and read Sections 9010 right away, since these sections are practically independent of 38

\section{The Gersten-Sherman Transformation}

To prove Theorem (2.12), we need to compare and/or identify various K-groups. The relevant definitions and properties of these K-groups are recalled below. The (so-called) Gersten-Sherman transformation is our most valuable tool for comparing $\mathrm{K}$-groups, and the main part of this section is devoted to this natural transformation. Readers who are familiar with K-theory may skip this section altogether.

In the following, the Grothendieck group functor is denoted by G.

(3.1) Let $A$ be a unital ring.

The classical $\mathrm{K}_{0}$-group of $A$ is defined as $\mathrm{K}_{0}^{\mathrm{C}}(A)=\mathrm{G}(\operatorname{proj} A)$, that is, the Grothendieck group of the category of finitely generated projective $A$-modules.

The classical $\mathrm{K}_{1}$-group of $A$ is defined as $\mathrm{K}_{1}^{\mathrm{C}}(A)=\mathrm{GL}(A)_{\mathrm{ab}}$, that is, the abelianization of the infinite (or stable) general linear group; see e.g. Bass [7, chap. V].

(3.2) Let $\mathcal{C}$ be any category. Its loop category $\Omega \mathcal{C}$ is the category whose objects are pairs $(C, \alpha)$ with $C \in \mathcal{C}$ and $\alpha \in \operatorname{Aut}_{\mathcal{C}}(C)$. A morphism $(C, \alpha) \rightarrow\left(C^{\prime}, \alpha^{\prime}\right)$ in $\Omega \mathcal{C}$ is 
a commutative diagram in $\mathcal{C}$,

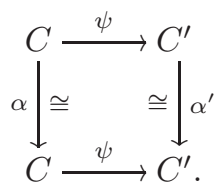

(3.3) Let $\mathcal{C}$ be a skeletally small exact category. Its loop category $\Omega \mathcal{C}$ is also skeletally small, and it inherits a natural exact structure from $\mathcal{C}$. Bass' $\mathrm{K}_{1}$-group (also called Bass' universal determinant group) of $\mathcal{C}$, which we denote by $\mathrm{K}_{1}^{\mathrm{B}}(\mathcal{C})$, is the Grothendieck group of $\Omega \mathcal{C}$, that is $\mathrm{G}(\Omega \mathcal{C})$, modulo the subgroup generated by all elements of the form

$$
(C, \alpha)+(C, \beta)-(C, \alpha \beta),
$$

where $C \in \mathcal{C}$ and $\alpha, \beta \in \operatorname{Aut}_{\mathcal{C}}(C)$; see the book of Bass [7, chap. VIII $\left.\S 1\right]$ or Rosenberg [25, def. 3.1.6]. For $(C, \alpha)$ in $\Omega \mathcal{C}$ we denote by $[C, \alpha]$ its image in $\mathrm{K}_{1}^{\mathrm{B}}(\mathcal{C})$.

(3.4) For every $C$ in $\mathcal{C}$ one has $\left[C, 1_{C}\right]+\left[C, 1_{C}\right]=\left[C, 1_{C} 1_{C}\right]=\left[C, 1_{C}\right]$ in $\mathrm{K}_{1}^{\mathrm{B}}(\mathcal{C})$. Consequently, $\left[C, 1_{C}\right]$ is the neutral element in $\mathrm{K}_{1}^{\mathrm{B}}(\mathcal{C})$.

(3.5) For a unital ring $A$ there is by [25, thm. 3.1.7] a natural isomorphism,

$$
\eta_{A}: \mathrm{K}_{1}^{\mathrm{C}}(A) \stackrel{\cong}{\longrightarrow} \mathrm{K}_{1}^{\mathrm{B}}(\operatorname{proj} A) \text {. }
$$

The isomorphism $\eta_{A}$ maps $\xi \in \mathrm{GL}_{n}(A)$, to the class $\left[A^{n}, \xi\right] \in \mathrm{K}_{1}^{\mathrm{B}}(\operatorname{proj} A)$. Here $\xi$ is viewed as an automorphism of the row space $A^{n}$ (a free left $A$-module), that is, $\xi$ acts by multiplication from the right.

The inverse map $\eta_{A}^{-1}$ acts as follows. Let $[P, \alpha]$ be in $\mathrm{K}_{1}^{\mathrm{B}}(\operatorname{proj} A)$. Choose any $Q$ in $\operatorname{proj} A$ and any isomorphism $\psi: P \oplus Q \rightarrow A^{n}$ with $n \in \mathbb{N}$. In $\mathrm{K}_{1}^{\mathrm{B}}(\operatorname{proj} A)$ one has

$$
[P, \alpha]=[P, \alpha]+\left[Q, 1_{Q}\right]=\left[P \oplus Q, \alpha \oplus 1_{Q}\right]=\left[A^{n}, \psi\left(\alpha \oplus 1_{Q}\right) \psi^{-1}\right] .
$$

The automorphism $\psi\left(\alpha \oplus 1_{Q}\right) \psi^{-1}$ of (the row space) $A^{n}$ can be identified with a matrix in $\beta \in \mathrm{GL}_{n}(A)$. The action of $\eta_{A}^{-1}$ on $[P, \alpha]$ is now $\beta$ 's image in $\mathrm{K}_{1}^{\mathrm{C}}(A)$.

(3.6) Quillen defines in [24] functors $\mathrm{K}_{n}^{\mathrm{Q}}$ from the category of skeletally small exact categories to the category of abelian groups. More precisely, $\mathrm{K}_{n}^{\mathrm{Q}}(\mathcal{C})=\pi_{n+1}(\mathrm{BQ} \mathcal{C}, 0)$ where Q is Quillen's Q-construction and B denotes the classifying space.

The functor $\mathrm{K}_{0}^{\mathrm{Q}}$ is naturally isomorphic to the Grothendieck group functor $\mathrm{G}$; see 24, $\S 2$ thm. 1]. For a $\operatorname{ring} A$ there is a natural isomorphism $\mathrm{K}_{1}^{\mathrm{Q}}(\operatorname{proj} A) \cong \mathrm{K}_{1}^{\mathrm{C}}(A)$; see for example Srinivas [27, cor. (2.6) and thm. (5.1)].

Gersten sketches in [17, §5] the construction of a natural transformation $\zeta: \mathrm{K}_{1}^{\mathrm{B}} \rightarrow$ $\mathrm{K}_{1}^{\mathrm{Q}}$ of functors on the category of skeletally small exact categories. The details of this construction were later given by Sherman [26, §3], and for this reason we refer to $\zeta$ as the Gersten-Sherman transformation 11. Examples due to Gersten and Murthy [17, prop. 5.1 and 5.2] show that for a general skeletally small exact category $\mathcal{C}$, the homomorphism $\zeta_{\mathcal{C}}: \mathrm{K}_{1}^{\mathrm{B}}(\mathcal{C}) \rightarrow \mathrm{K}_{1}^{\mathrm{Q}}(\mathcal{C})$ is neither injective nor surjective. For the exact category proj $A$, where $A$ is a ring, it is known that $\mathrm{K}_{1}^{\mathrm{B}}(\operatorname{proj} A)$ and $\mathrm{K}_{1}^{\mathrm{Q}}(\operatorname{proj} A)$ are isomorphic, indeed, they are both isomorphic to the classical K-group $\mathrm{K}_{1}^{\mathrm{C}}(A)$; see (3.5) and (3.6). Therefore, a natural question arises: is $\zeta_{\text {proj } A}$ an isomorphism? Sherman answers this question affirmatively in [26. pp. 231-232]; in fact, in loc. cit.

\footnotetext{
${ }^{1}$ In the papers by Gersten [17] and Sherman [26], the functor $K_{1}^{B}$ is denoted by $K_{1}^{\text {det }}$.
} 
Theorem 3.3 it is proved that $\zeta_{\mathcal{C}}$ is an isomorphism for every semisimple exact category, that is, an exact category in which every short exact sequence splits. We note these results of Gersten and Sherman for later use.

(3.7) Theorem. There exists a natural transformation $\zeta: \mathrm{K}_{1}^{\mathrm{B}} \rightarrow \mathrm{K}_{1}^{\mathrm{Q}}$, which we call the Gersten-Sherman transformation, of functors on the category of skeletally small exact categories such that $\zeta_{\operatorname{proj} A}: \mathrm{K}_{1}^{\mathrm{B}}(\operatorname{proj} A) \rightarrow \mathrm{K}_{1}^{\mathrm{Q}}(\operatorname{proj} A)$ is an isomorphism for every ring $A$.

We will also need the next result on the Gersten-Sherman transformation. Recall that a length category is an abelian category in which every object has finite length.

(3.8) Theorem. If $\mathcal{A}$ is a skeletally small length category with only finitely many simple objects (up to isomorphism), then $\zeta_{\mathcal{A}}: \mathrm{K}_{1}^{\mathrm{B}}(\mathcal{A}) \rightarrow \mathrm{K}_{1}^{\mathrm{Q}}(\mathcal{A})$ is an isomorphism.

Proof. We begin with a general observation. Given skeletally small exact categories $\mathcal{C}_{1}$ and $\mathcal{C}_{2}$, there are exact projection functors $p_{j}: \mathcal{C}_{1} \times \mathcal{C}_{2} \rightarrow \mathcal{C}_{j}(j=1,2)$. From the "elementary properties" of Quillen's K-groups listed in [24, \$2], it follows that the homomorphism $\left(\mathrm{K}_{1}^{\mathrm{Q}}\left(p_{1}\right), \mathrm{K}_{1}^{\mathrm{Q}}\left(p_{2}\right)\right): \mathrm{K}_{1}^{\mathrm{Q}}\left(\mathcal{C}_{1} \times \mathcal{C}_{2}\right) \rightarrow \mathrm{K}_{1}^{\mathrm{Q}}\left(\mathcal{C}_{1}\right) \oplus \mathrm{K}_{1}^{\mathrm{Q}}\left(\mathcal{C}_{2}\right)$ is an isomorphism. A similar argument shows that $\left(\mathrm{K}_{1}^{\mathrm{B}}\left(p_{1}\right), \mathrm{K}_{1}^{\mathrm{B}}\left(p_{2}\right)\right)$ is an isomorphism. Since $\zeta: \mathrm{K}_{1}^{\mathrm{B}} \rightarrow \mathrm{K}_{1}^{\mathrm{Q}}$ is a natural transformation, it follows that $\zeta_{\mathcal{C}_{1} \times \mathcal{C}_{2}}$ is an isomorphism if and only if $\zeta_{\mathcal{C}_{1}}$ and $\zeta_{\mathcal{C}_{2}}$ are isomorphisms.

Denote by $\mathcal{A}_{\mathrm{ss}}$ the full subcategory of $\mathcal{A}$ consisting of all semisimple objects. Note that $\mathcal{A}_{\mathrm{ss}}$ is a Serre subcategory of $\mathcal{A}$, and hence $\mathcal{A}_{\mathrm{ss}}$ is itself an abelian category. Let $i: \mathcal{A}_{\mathrm{ss}} \hookrightarrow \mathcal{A}$ be the (exact) inclusion and consider the commutative diagram,

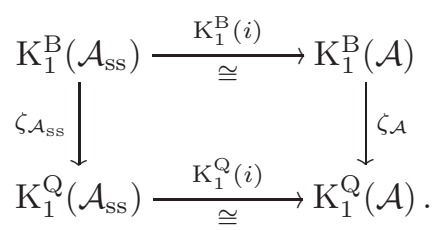

Since $\mathcal{A}$ is a length category, Bass' and Quillen's devissage theorems [7, VIII $§ 3$ thm. $(3.4)(\mathrm{a})]$ and $\left[24, \S 5\right.$ thm. 4] show that $\mathrm{K}_{1}^{\mathrm{B}}(i)$ and $\mathrm{K}_{1}^{\mathrm{Q}}(i)$ are isomorphisms. Hence, it suffices to argue that $\zeta_{\mathcal{A}_{\mathrm{ss}}}$ is an isomorphism. By assumption there is a finite set $\left\{S_{1}, \ldots, S_{n}\right\}$ of representatives of the isomorphism classes of simple objects in $\mathcal{A}$. Note that every object $A$ in $\mathcal{A}_{\mathrm{ss}}$ has unique decomposition $A=S_{1}^{a_{1}} \oplus \cdots \oplus S_{n}^{a_{n}}$ where $a_{1}, \ldots, a_{n} \in \mathbb{N}_{0}$; we used here the assumption that $A$ has finite length to conclude that the cardinal numbers $a_{i}$ must be finite. Since one has $\operatorname{Hom}_{\mathcal{A}}\left(S_{i}, S_{j}\right)=0$ for $i \neq j$, it follows that there is an equivalence of abelian categories,

$$
\mathcal{A}_{\mathrm{ss}} \simeq\left(\operatorname{add} S_{1}\right) \times \cdots \times\left(\operatorname{add} S_{n}\right) .
$$

Consider the ring $D_{i}=\operatorname{End}_{\mathcal{A}}\left(S_{i}\right)^{\text {op }}$. As $S_{i}$ is simple, Schur's lemma gives that $D_{i}$ is a division ring. It easy to see that the functor $\operatorname{Hom}_{\mathcal{A}}\left(S_{i},-\right): \mathcal{A} \rightarrow \operatorname{Mod} D_{i}$ induces an equivalence add $S_{i} \simeq \operatorname{proj} D_{i}$. By Theorem (3.7) the maps $\zeta_{\text {proj } D_{1}}, \ldots, \zeta_{\text {proj }} D_{n}$ are isomorphisms, so it follows from the equivalence above, and the general observation in the beginning af the proof, that $\zeta_{\mathcal{A}_{\mathrm{ss}}}$ is an isomorphism, as desired.

Note that in this section, superscripts "C" (for classical), "B" (for Bass), and "Q" (for Quillen) have been used to distinguish between various K-groups. In the rest of the paper, K-groups without superscripts refer to Quillen's K-groups. 


\section{Coherent Pairs}

We recall a few results and notions from the paper [4 by Auslander and Reiten which are central in the proof of our main Theorem (2.12). Throughout this section, $\mathcal{A}$ denotes a skeletally small additive category.

(4.1) Definition. A pseudo (or weak) kernel of a morphism $g: A \rightarrow A^{\prime}$ in $\mathcal{A}$ is a morphism $f: A^{\prime \prime} \rightarrow A$ in $\mathcal{A}$ such that $g f=0$, and which satisfies that every diagram in $\mathcal{A}$ as below can be completed (but not necessarily in a unique way).

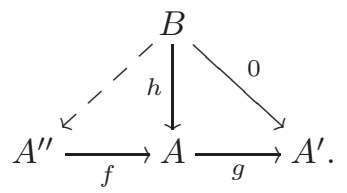

We say that $\mathcal{A}$ has pseudo kernels if every morphism in $\mathcal{A}$ has a pseudo kernel.

(4.2) Observation. Let $\mathcal{A}$ be a full additive subcategory of an abelian category $\mathcal{M}$. An $\mathcal{A}$-precover of an object $M \in \mathcal{M}$ is a morphism $u: A \rightarrow M$ with $A \in \mathcal{A}$ with the property that for every morphism $u^{\prime}: A^{\prime} \rightarrow M$ with $A^{\prime} \in \mathcal{A}$ there exists a (not necessarily unique) morphism $v: A^{\prime} \rightarrow A$ such that $u v=u^{\prime}$. Following [13, def. 5.1.1] we say that $\mathcal{A}$ is precovering (or contravariantly finite) in $\mathcal{M}$ if every object $M \in \mathcal{M}$ has an $\mathcal{A}$-precover. In this case, $\mathcal{A}$ has pseudo kernels. Indeed, if $i: K \rightarrow A$ is the kernel in $\mathcal{M}$ of $g: A \rightarrow A^{\prime}$ in $\mathcal{A}$, and if $f: A^{\prime \prime} \rightarrow K$ is an $\mathcal{A}$-precover of $K$, then if: $A^{\prime \prime} \rightarrow A$ is a pseudo kernel of $g$.

(4.3) Definition. Let $\mathcal{B}$ be a full additive subcategory of $\mathcal{A}$. Auslander and Reiten [4] call $(\mathcal{A}, \mathcal{B})$ a coherent pair if $\mathcal{A}$ has pseudo kernels in the sense of Definition (4.1), and $\mathcal{B}$ is precovering in $\mathcal{A}$.

If $(\mathcal{A}, \mathcal{B})$ is a coherent pair then also $\mathcal{B}$ has pseudo kernels by [4, prop. 1.4(a)].

(4.4) Definition. Write $\operatorname{Mod} \mathcal{A}$ for the abelian category of additive contravariant functors $\mathcal{A} \rightarrow \mathrm{Ab}$, where $\mathrm{Ab}$ is the category of abelian groups. Denote by mod $\mathcal{A}$ the full subcategory of $\operatorname{Mod} \mathcal{A}$ consisting of finitely presented functors.

(4.5) If the category $\mathcal{A}$ has pseudo kernels then $\bmod \mathcal{A}$ is abelian, and the inclusion functor $\bmod \mathcal{A} \rightarrow \operatorname{Mod} \mathcal{A}$ is exact, see [4, prop. 1.3].

If $(\mathcal{A}, \mathcal{B})$ is a coherent pair, see (4.3), then the exact restriction $\operatorname{Mod} \mathcal{A} \rightarrow \operatorname{Mod} \mathcal{B}$ $\operatorname{maps} \bmod \mathcal{A}$ to $\bmod \mathcal{B}$ by 4 , prop. $1.4(\mathrm{~b})]$. In this case, there are functors,

$$
\operatorname{Ker} r \stackrel{i}{\longrightarrow} \bmod \mathcal{A} \stackrel{r}{\longrightarrow} \bmod \mathcal{B}
$$

where $r$ is the restriction and $i$ the inclusion functor. The kernel of $r$, that is,

$$
\operatorname{Ker} r=\{F \in \bmod \mathcal{A} \mid F(B)=0 \text { for all } B \in \mathcal{B}\},
$$

is a Serre subcategory of the abelian category $\bmod \mathcal{A}$. The quotient $(\bmod \mathcal{A}) /(\operatorname{Ker} r)$, in the sense of Gabriel [16], is equivalent to the category $\bmod \mathcal{B}$, and the canonical functor $\bmod \mathcal{A} \rightarrow(\bmod \mathcal{A}) /(\operatorname{Ker} r)$ may be identified with $r$. These assertions are proved in [4, prop. 1.5]. Therefore (4.5.1) induces by Quillen's localization theorem 
[24. $\S 5$ thm. 5] a long exact sequence of K-groups,

$$
\begin{aligned}
& \cdots \longrightarrow \mathrm{K}_{n}(\operatorname{Ker} r) \stackrel{\mathrm{K}_{n}(i)}{\longrightarrow} \mathrm{K}_{n}(\bmod \mathcal{A}) \stackrel{\mathrm{K}_{n}(r)}{\longrightarrow} \mathrm{K}_{n}(\bmod \mathcal{B}) \longrightarrow \cdots \\
& \cdots \longrightarrow \mathrm{K}_{0}(\operatorname{Ker} r) \stackrel{\mathrm{K}_{0}(i)}{\longrightarrow} \mathrm{K}_{0}(\bmod \mathcal{A}) \stackrel{\mathrm{K}_{0}(r)}{\longrightarrow} \mathrm{K}_{0}(\bmod \mathcal{B}) \longrightarrow 0
\end{aligned}
$$

\section{Semilocal Rings}

A ring $A$ is semilocal if $A / \mathrm{J}(A)$ is semisimple. Here $\mathrm{J}(A)$ is the Jacobson radical of $A$. If $A$ is commutative then this definition is equivalent to $A$ having only finitely many maximal ideals; see Lam [20, prop. (20.2)].

(5.1) Lemma. Let $R$ be a commutative noetherian semilocal ring, and let $M \neq 0$ be a finitely generated $R$-module. Then the ring $\operatorname{End}_{R}(M)$ is semilocal.

Proof. As $R$ is commutative and noetherian, $\operatorname{End}_{R}(M)$ is a module-finite $R$-algebra. Since $R$ is semilocal, the assertion now follows from [20, prop. (20.6)].

(5.2) Denote by $A^{*}$ the group of units in a ring $A$, and let $\vartheta_{A}: A^{*} \rightarrow \mathrm{K}_{1}^{\mathrm{C}}(A)$ be the composite of the group homomorphisms,

$$
A^{*} \cong \mathrm{GL}_{1}(A) \hookrightarrow \mathrm{GL}(A) \rightarrow \mathrm{GL}(A)_{\mathrm{ab}}=\mathrm{K}_{1}^{\mathrm{C}}(A) .
$$

Some authors refer to $\vartheta_{A}$ as the Whitehead determinant. If $A$ is semilocal, then $\vartheta_{A}$ is surjective by Bass [7, $\mathrm{V} \S 9$ thm. (9.1)]. As the group $\mathrm{K}_{1}^{\mathrm{C}}(A)$ is abelian one has $\left[A^{*}, A^{*}\right] \subseteq \operatorname{Ker} \vartheta_{A}$, and we write $\theta_{A}: A_{\mathrm{ab}}^{*} \rightarrow \mathrm{K}_{1}^{\mathrm{C}}(A)$ for the induced homomorphism.

Vaserstein [28, showed that the inclusion $\left[A^{*}, A^{*}\right] \subseteq \operatorname{Ker} \vartheta_{A}$ is strict for the semilocal ring $A=\mathrm{M}_{2}\left(\mathbb{F}_{2}\right)$ where $\mathbb{F}_{2}$ is the field with two elements. In 28, thm. 3.6(a)] it is shown that if $A$ is semilocal, then $\operatorname{Ker} \vartheta_{A}$ is the subgroup of $A^{*}$ generated by elements of the form $(1+a b)(1+b a)^{-1}$ where $a, b \in A$ and $1+a b \in A^{*}$.

If $A$ is semilocal, that is, $A / \mathrm{J}(A)$ is semisimple, then by the Artin-Wedderburn theorem there is an isomorphism of rings,

$$
A / \mathrm{J}(A) \cong \mathrm{M}_{n_{1}}\left(D_{1}\right) \times \cdots \times \mathrm{M}_{n_{t}}\left(D_{t}\right),
$$

where $D_{1}, \ldots, D_{t}$ are division rings, and $n_{1}, \ldots, n_{t}$ are natural numbers all of which are uniquely determined by $A$. The next result is due to Vaserstein [29, thm. 2].

(5.3) Theorem. Let $A$ be semilocal and write $A / \mathrm{J}(A) \cong \mathrm{M}_{n_{1}}\left(D_{1}\right) \times \cdots \times \mathrm{M}_{n_{t}}\left(D_{t}\right)$. If none of the $\mathrm{M}_{n_{i}}\left(D_{i}\right)$ 's is $\mathrm{M}_{2}\left(\mathbb{F}_{2}\right)$, and at most one of the $\mathrm{M}_{n_{i}}\left(D_{i}\right)$ 's is $\mathrm{M}_{1}\left(\mathbb{F}_{2}\right)=\mathbb{F}_{2}$ then one has $\operatorname{Ker} \vartheta_{A}=\left[A^{*}, A^{*}\right]$. In particular, $\vartheta_{A}$ induces an isomorphism,

$$
\theta_{A}: A_{\mathrm{ab}}^{*} \stackrel{\cong}{\longrightarrow} \mathrm{K}_{1}^{\mathrm{C}}(A) \text {. }
$$

(5.4) Remark. Note that if $A$ is a semilocal ring which is an algebra over a field $k$ with characteristic $\neq 2$, then the hypothesis in Theorem (5.3) is satisfied.

If $A$ is a commutative semilocal ring, then $\operatorname{Ker} \vartheta_{A}$ and the commutator subgroup $\left[A^{*}, A^{*}\right]=\{1\}$ are identical, i.e. the surjective homomorphism $\vartheta_{A}=\theta_{A}: A^{*} \rightarrow \mathrm{K}_{1}^{\mathrm{C}}(A)$ is an isomorphism. Indeed, the determinant homomorphisms $\operatorname{det}_{n}: \mathrm{GL}_{n}(A) \rightarrow A^{*}$ induce a homomorphism $\operatorname{det}_{A}: \mathrm{K}_{1}^{\mathrm{C}}(A) \rightarrow A^{*}$ that evidently satisfies $\operatorname{det}_{A} \theta_{A}=1_{A^{*}}$. Since $\theta_{A}$ is surjective, it follows that $\theta_{A}$ is an isomorphism with $\theta_{A}^{-1}=\operatorname{det}_{A}$. 
(5.5) Definition. Let $A$ be a ring for which the homomorphism $\theta_{A}: A_{\mathrm{ab}}^{*} \rightarrow \mathrm{K}_{1}^{\mathrm{C}}(A)$ from (5.2) is an isomorphism; for example, $A$ could be a commutative semilocal ring or a noncommutative semilocal ring satisfying the assumptions in Theorem (5.3). The inverse $\theta_{A}^{-1}$ is denoted by $\operatorname{det}_{A}$, and we call it the generalized determinant.

(5.6) Remark. Let $\xi$ be an $m \times n$ and let $\chi$ be an $n \times p$ matrix with entries in a ring $A$. Denote by "." the product $\mathrm{M}_{m \times n}\left(A^{\mathrm{op}}\right) \times \mathrm{M}_{n \times p}\left(A^{\mathrm{op}}\right) \rightarrow \mathrm{M}_{m \times p}\left(A^{\mathrm{op}}\right)$. Then

$$
(\xi \cdot \chi)^{T}=\chi^{T} \xi^{T},
$$

where $\chi^{T} \xi^{T}$ is computed using the product $\mathrm{M}_{p \times n}(A) \times \mathrm{M}_{n \times m}(A) \rightarrow \mathrm{M}_{p \times m}(A)$. Thus, transposition $(-)^{T}: \mathrm{GL}_{n}\left(A^{\mathrm{op}}\right) \rightarrow \mathrm{GL}_{n}(A)$ is an anti-isomorphism (this is also noted in [7, V§7]), which induces an isomorphism $(-)^{T}: \mathrm{K}_{1}^{\mathrm{C}}\left(A^{\mathrm{op}}\right) \rightarrow \mathrm{K}_{1}^{\mathrm{C}}(A)$.

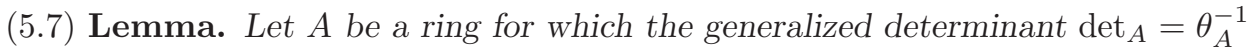
exists; cf. Definition (5.5). For every invertible matrix $\xi$ with entries in $A$ one has an equality $\operatorname{det}_{A^{\text {op }}}\left(\xi^{T}\right)=\operatorname{det}_{A}(\xi)$ in the abelian group $\left(A^{\mathrm{op}}\right)_{\mathrm{ab}}^{*}=A_{\mathrm{ab}}^{*}$.

Proof. Clearly, there is a commutative diagram,

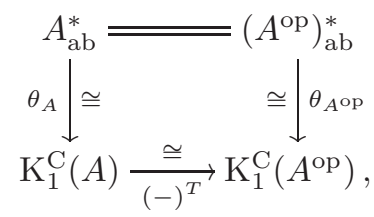

It follows that one has $\theta_{A^{\text {op }}}^{-1} \circ(-)^{T}=\theta_{A}^{-1}$, that is, $\operatorname{det}_{A^{\text {op }}} \circ(-)^{T}=\operatorname{det}_{A}$.

\section{Some Useful Functors}

Throughout this section, $A$ is a ring and $M$ is a fixed left $A$-module. We denote by $E=\operatorname{End}_{A}(M)$ the endomorphism ring of $M$. Note that $M={ }_{A, E} M$ has a natural left- $A$-left- $E$-bimodule structure.

(6.1) There is a pair of adjoint functors,

$$
\operatorname{Mod} A \underset{-\otimes_{E} M}{\stackrel{\operatorname{Hom}_{A}(M,-)}{\rightleftarrows}} \operatorname{Mod}\left(E^{\mathrm{op}}\right) .
$$

It is easily seen that they restrict to a pair of quasi-inverse equivalences,

$$
\operatorname{add}_{A} M \underset{-\otimes_{E} M}{\stackrel{\operatorname{Hom}_{A}(M,-)}{\simeq}} \operatorname{proj}\left(E^{\mathrm{op}}\right) .
$$

Auslander referred to this phenomenon as projectivization; see [6. I $\$ 2]$.

Let $F \in \operatorname{Mod}\left(\operatorname{add}_{A} M\right)$, that is, $F: \operatorname{add}_{A} M \rightarrow$ Ab is a contravariant additive functor, see Definition (4.4). The compatible $E$-module structure on the given $A$-module $M$ induces an $E^{\mathrm{op}}$-module structure on the abelian group $F M$ which is given by $z \alpha=(F \alpha)(z)$ for $\alpha \in E$ and $z \in F M$.

(6.2) Proposition. There are quasi-inverse equivalences of abelian categories,

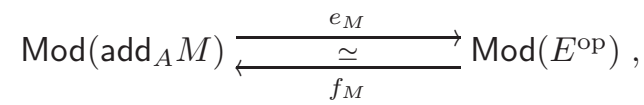


where $e_{M}$ (evaluation) and $f_{M}$ (functorfication) are defined as follows,

$$
e_{M}(F)=F M \quad \text { and } \quad f_{M}(Z)=\left.Z \otimes_{E} \operatorname{Hom}_{A}(-, M)\right|_{\operatorname{add}_{A} M},
$$

for $F$ in $\operatorname{Mod}\left(\operatorname{add}_{A} M\right)$ and $Z$ in $\operatorname{Mod}\left(E^{\mathrm{op}}\right)$. They restrict to quasi-inverse equivalences between categories of finitely presented objects,

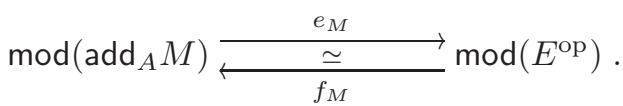

Proof. For $Z$ in $\operatorname{Mod}\left(E^{\text {op }}\right)$ the canonical isomorphism

$$
Z \stackrel{\cong}{\longrightarrow} Z \otimes_{E} E=Z \otimes_{E} \operatorname{Hom}_{A}(M, M)=e_{M} f_{M}(Z)
$$

is natural in $Z$. Thus, the functors $\operatorname{id}_{\operatorname{Mod}\left(E^{\mathrm{op}}\right)}$ and $e_{M} f_{M}$ are naturally isomorphic. For $F$ in $\operatorname{Mod}\left(\operatorname{add}_{A} M\right)$ there is a natural transformation,

$$
f_{M} e_{M}(F)=\left.F M \otimes_{E} \operatorname{Hom}_{A}(-, M)\right|_{\operatorname{add}_{A} M} \stackrel{\delta}{\longrightarrow} F ;
$$

for $X$ in $\operatorname{add}_{A} M$ the homomorphism $\delta_{X}: F M \otimes_{E} \operatorname{Hom}_{A}(X, M) \rightarrow F X$ is given by $z \otimes \psi \mapsto(F \psi)(z)$. Note that $\delta_{M}$ is an isomorphism as it may be identified with the canonical isomorphism $F M \otimes_{E} E \stackrel{\cong}{\longrightarrow} F M$ in Ab. As the functors in (6.2.1) are additive, it follows that $\delta_{X}$ is an isomorphism for every $X \in \operatorname{add}_{A} M$, that is, $\delta$ is a natural isomorphism. Since (6.2.1) is natural in $F$, the functors $f_{M} e_{M}$ and $\operatorname{id}_{\operatorname{Mod}\left(\operatorname{add}_{A} M\right)}$ are naturally isomorphic.

It is straightforward to verify that the functors $e_{M}$ and $f_{M}$ map finitely presented objects to finitely presented objects.

(6.3) Observation. In the case $M=A$ one has $E=\operatorname{End}_{A}(M)=A^{\mathrm{op}}$, and therefore Proposition (6.2) yields an equivalence $f_{A}: \bmod A \rightarrow \bmod (\operatorname{proj} A)$ given by

$$
\left.X \longmapsto X \otimes_{A^{\mathrm{op}}} \operatorname{Hom}_{A}(-, A)\right|_{\operatorname{proj} A} .
$$

It is easily seen that the functor $f_{A}$ is naturally isomorphic to the functor given by

$$
\left.X \longmapsto \operatorname{Hom}_{A}(-, X)\right|_{\operatorname{proj} A} .
$$

We will usually identify $f_{A}$ with this functor.

(6.4) Definition. The functor $y_{M}: \operatorname{add}_{A} M \rightarrow \bmod \left(\operatorname{add}_{A} M\right)$ which for $X \in \operatorname{add}_{A} M$ is given by $y_{M}(X)=\left.\operatorname{Hom}_{A}(-, X)\right|_{\operatorname{add}_{A} M}$ is called the Yoneda functor.

Let $\mathcal{A}$ be a full additive subcategory of an abelian category $\mathcal{M}$. If $\mathcal{A}$ is closed under extensions in $\mathcal{M}$, then $\mathcal{A}$ has a natural induced exact structure. However, one can always equip $\mathcal{A}$ with the trivial exact structure. In this structure, the "exact sequences" (somtimes called conflations) are only the split exact ones. When viewing $\mathcal{A}$ as an exact category with the trivial exact structure, we denote it $\mathcal{A}_{0}$.

(6.5) Lemma. Assume that $A$ is commutative and noetherian and let $M \in \bmod A$. Set $E=\operatorname{End}_{A}(M)$ and assume that $E^{\mathrm{op}}$ has finite global dimension. For the exact Yoneda functor $y_{M}:\left(\operatorname{add}_{A} M\right)_{0} \rightarrow \bmod \left(\operatorname{add}_{A} M\right)$, see 6.4), the homomorphisms $\mathrm{K}_{n}\left(y_{M}\right)$, where $n \geqslant 0$, and $\mathrm{K}_{1}^{\mathrm{B}}\left(y_{M}\right)$ are isomorphisms. 
Proof. By application of $\mathrm{K}_{n}$ to the commutative diagram,

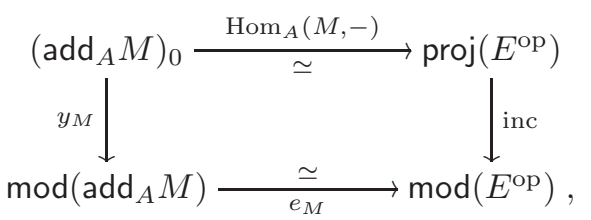

it follows that $\mathrm{K}_{n}\left(y_{M}\right)$ is an isomorphism if and only if $\mathrm{K}_{n}$ (inc) is an isomorphism. The latter holds by Quillen's resolution theorem [24, $\S 4$ thm. 3], since $E^{\mathrm{op}}$ has finite global dimension. A similar argument shows that $\mathrm{K}_{1}^{\mathrm{B}}\left(y_{M}\right)$ is an isomorphism. This time one needs to apply Bass' resolution theorem; see [7, VIII§4 thm. (4.6)].

Since $\mathrm{K}_{0}$ may be identified with the Grothendieck group functor, cf. (3.6), the following result is well-known. In any case, it is straightforward to verify.

(6.6) Lemma. Assume that $\bmod A$ is Krull-Schmidt. Let $N=N_{1}^{n_{1}} \oplus \cdots \oplus N_{s}^{n_{s}}$ be a finitely generated $A$-module, where $N_{1}, \ldots, N_{s}$ are non-isomorphic indecomposable $A$-modules and $n_{1}, \ldots, n_{s}>0$. The homomorphism of abelian groups,

$$
\psi_{N}: \mathbb{Z} N_{1} \oplus \cdots \oplus \mathbb{Z} N_{s} \longrightarrow \mathrm{K}_{0}\left(\left(\operatorname{add}_{A} N\right)_{0}\right),
$$

given by $N_{j} \mapsto\left[N_{j}\right]$, is an isomorphism.

\section{The Abelian Category $\mathcal{Y}$}

By the assumptions in Setup (2.1), the ground ring $R$ has a dualizing module. It follows from Auslander and Buchweitz [3, thm. A] that MCM $R$ is precovering in $\bmod R$. Actually, in our case MCM $R$ equals $\operatorname{add}_{R} M$ for some finitely generated $R$-module $M$ (a representation generator), and it is easily seen that every category of this form is precovering in $\bmod R$. By Observation (4.2) we have a coherent pair $(\mathrm{MCM} R, \operatorname{proj} R$ ), which by (4.5) yields a Gabriel localization sequence,

$$
\mathcal{Y}=\operatorname{Ker} r \stackrel{i}{\longrightarrow} \bmod (\mathrm{MCM} R) \stackrel{r}{\longrightarrow} \bmod (\operatorname{proj} R) .
$$

Here $r$ is the restriction functor, $\mathcal{Y}=\operatorname{Ker} r$, and $i$ is the inclusion. Since an additive functor vanishes on proj $R$ if and only if it vanishes on $R$, one has

$$
\mathcal{Y}=\{F \in \bmod (\mathrm{MCM} R) \mid F(R)=0\} .
$$

The following two results about the abelian category $\mathcal{Y}$ are due to Yoshino. The first result is [32, (13.7.4)]; the second is (proofs of) [32, lem. (4.12) and prop. (4.13)].

(7.1) Theorem. Every object in $\mathcal{Y}$ has finite length, i.e. $\mathcal{Y}$ is a length category.

(7.2) Theorem. Consider for $1 \leqslant j \leqslant t$ the Auslander-Reiten sequence (2.1.2) ending in $M_{j}$. The functor $F_{j}$, defined by the following exact sequence in $\bmod (\mathrm{MCM} R)$,

$$
0 \longrightarrow \operatorname{Hom}_{R}\left(-, \tau\left(M_{j}\right)\right) \longrightarrow \operatorname{Hom}_{R}\left(-, X_{j}\right) \longrightarrow \operatorname{Hom}_{R}\left(-, M_{j}\right) \longrightarrow F_{j} \longrightarrow 0,
$$

is a simple object in $\mathcal{Y}$. Conversely, every simple functor in $\mathcal{Y}$ is naturally isomorphic to $F_{j}$ for some $1 \leqslant j \leqslant t$.

(7.3) Proposition. Let $i: \mathcal{Y} \rightarrow \bmod (\mathrm{MCM} R$ ) be the inclusion functor from (7.0.1) and let $\Upsilon: \mathbb{Z}^{t} \rightarrow \mathbb{Z}^{t+1}$ be the Auslander-Reiten homomorphism; see Definition (2.3). The homomorphisms $\mathrm{K}_{0}(i)$ and $\Upsilon$ are isomorphic. 
Proof. We claim that the following diagram of abelian groups is commutative,

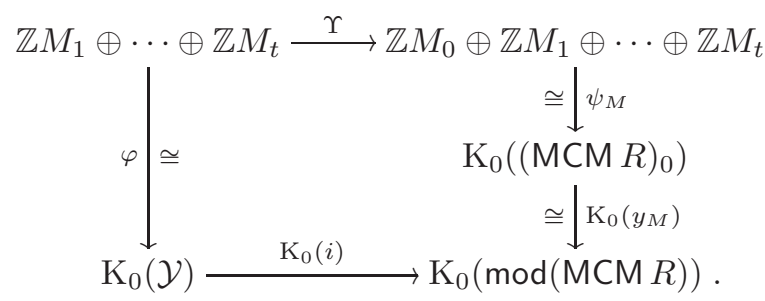

The homomorphism $\varphi$ is defined by $M_{j} \mapsto\left[F_{j}\right]$ where $F_{j} \in \mathcal{Y}$ is described in (7.2). From Theorems (7.1) and (7.2) and the proof of Rosenberg [25, thm. 3.1.8(1)] (or the proof of Theorem (3.8) ), it follows that $\varphi$ is an isomorphism. The module $M$ is a representation generator for $\operatorname{MCM} R$, see (2.1), and $\psi_{M}$ is the isomorphism given in Lemma (6.6). Finally, $y_{M}$ is the Yoneda functor from Definition (6.4). By Leuschke [21, thm. 6] the ring $E^{\text {op }}$, where $E=\operatorname{End}_{R}(M)$, has finite global dimension, and thus Lemma (6.5) implies that $\mathrm{K}_{0}\left(y_{M}\right)$ is an isomorphism.

From the definitions of the relevant homomorphims, it is straightforward to see that the diagram is commutative; indeed, both $\mathrm{K}_{0}(i) \varphi$ and $\mathrm{K}_{0}\left(y_{M}\right) \psi_{M} \Upsilon$ map a generator $M_{j}$ to the element $\left[F_{j}\right] \in \mathrm{K}_{0}(\bmod (\mathrm{MCM} R))$.

\section{Proof of the Main Theorem}

Throughout this section, we fix the notation in Setup (2.1). Thus, $R$ is a commutative noetherian local Cohen-Macaulay ring satisfying conditions (2.1) (1)-(3), $M$ is any representation generator of $\mathrm{MCM} R$, and $E$ is its endomorphism ring.

We shall frequently make use of the Gabriel localization sequence (7.0.1), and $i$ and $r$ always denote the inclusion and the restriction functor in this sequence.

(8.1) Remark. Let $\mathcal{C}$ be an exact category. As in the paragraph preceding Lemma (6.5), we denote by $\mathcal{C}_{0}$ the category $\mathcal{C}$ equipped with the trivial exact structure. Note that the identity functor $\operatorname{id}_{\mathcal{C}}: \mathcal{C}_{0} \rightarrow \mathcal{C}$ is exact and the induced homomorphism $\mathrm{K}_{1}^{\mathrm{B}}\left(\operatorname{id}_{\mathcal{C}}\right): \mathrm{K}_{1}^{\mathrm{B}}\left(\mathcal{C}_{0}\right) \rightarrow \mathrm{K}_{1}^{\mathrm{B}}(\mathcal{C})$ is surjective, indeed, one has $\mathrm{K}_{1}^{\mathrm{B}}\left(\operatorname{id}_{\mathcal{C}}\right)([C, \alpha])=[C, \alpha]$.

(8.2) Lemma. Consider the restriction functor $r: \bmod (\mathrm{MCM} R) \rightarrow \bmod (\operatorname{proj} R)$ and identity functor $\operatorname{id}_{\mathrm{MCM} R}:(\mathrm{MCM} R)_{0} \rightarrow \mathrm{MCM} R$. The homomorphisms $\mathrm{K}_{1}^{\mathrm{B}}(r)$ and $\mathrm{K}_{1}^{\mathrm{B}}\left(\mathrm{id}_{\mathrm{MCM} R}\right)$ are isomorphic, in particular, $\mathrm{K}_{1}^{\mathrm{B}}(r)$ is surjective by Remark (8.1).

Proof. Consider the commutative diagram of exact categories and exact functors,

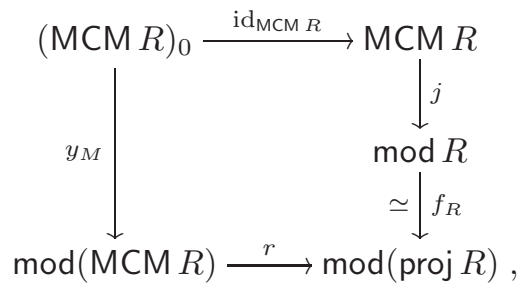

where $y_{M}$ is the Yoneda functor from Definition (6.4), $j$ is the inclusion, and $f_{R}$ is the equivalence from Observation (6.3). We will prove the lemma by arguing that the vertical functors induce isomorphisms on the level of $K_{1}^{B}$.

The ring $E^{\mathrm{op}}$ has finite global dimension by Leuschke [21, thm. 6], and hence Lemma (6.5) gives that that $\mathrm{K}_{1}^{\mathrm{B}}\left(y_{M}\right)$ is an isomorphism. Since $f_{R}$ is an equivalence, 
$\mathrm{K}_{1}^{\mathrm{B}}\left(f_{R}\right)$ is obviously an isomorphism. To argue that $\mathrm{K}_{1}^{\mathrm{B}}(j)$ is an isomorphism, we apply Bass' resolution theorem [25, thm. 3.1.14]. We must check that the subcategory MCM $R$ of $\bmod R$ satisfies conditions (1)-(3) in loc. cit. Condition (1) follows as MCM $R$ is precovering in $\bmod R$. As $R$ is Cohen-Macaulay, every module in $\bmod R$ has a resolution of finite length by modules in MCM $R$, see [32, prop. (1.4)]; thus condition (2) holds. Condition (3) requires that MCM $R$ is closed under kernels of epimorphisms; this is well-known from e.g. [32, prop. (1.3)].

Next we show some results on the Gersten-Sherman transformation; see Sect. 3

(8.3) Lemma. $\zeta_{\mathcal{C}}: \mathrm{K}_{1}^{\mathrm{B}}(\mathcal{C}) \rightarrow \mathrm{K}_{1}(\mathcal{C})$ is an isomorphism for $\mathcal{C}=\bmod (\mathrm{MCM} R)$.

Proof. As $\zeta$ is a natural transformation, there is a commutative diagram,

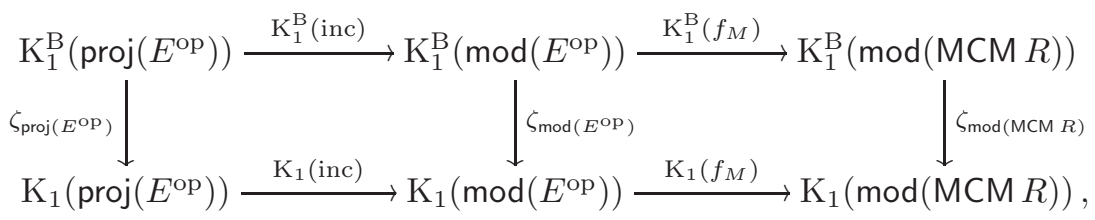

where $f_{M}: \bmod \left(E^{\mathrm{op}}\right) \rightarrow \bmod (\mathrm{MCM} R)$ is the equivalence from Proposition (6.2) and inc is the inclusion of $\operatorname{proj}\left(E^{\mathrm{op}}\right)$ into $\bmod \left(E^{\mathrm{op}}\right)$.

From Leuschke [21, thm. 6], the noetherian ring $E^{\mathrm{op}}$ has finite global dimension. Hence Bass' and Quillen's resolution theorems, [7, VIII $\S 4$ thm. (4.6)] (see also Rosenberg [25, thm. 3.1.14]) and [24, §4 thm. 3], imply that $\mathrm{K}_{1}^{\mathrm{B}}$ (inc) and $\mathrm{K}_{1}$ (inc) are isomorphisms. Since $f_{M}$ is an equivalence, $\mathrm{K}_{1}^{\mathrm{B}}\left(f_{M}\right)$ and $\mathrm{K}_{1}\left(f_{M}\right)$ are isomorphisms as well. Consequently, $\zeta_{\bmod (\operatorname{MCM} R)}$ is an isomorphism if and only if $\zeta_{\operatorname{proj}\left(E^{\mathrm{op}}\right)}$ is an isomorphism, and the latter holds by Theorem (3.7).

The goal is to compute Quillen's K-group $\mathrm{K}_{1}(\bmod R)$ for the $\operatorname{ring} R$ in question. For our proof of Theorem (2.12), it is crucial that this group can be naturally identified with Bass' K-group $\mathrm{K}_{1}^{\mathrm{B}}(\bmod R)$. To put Proposition (8.4) in perspective, we remind the reader that the Gersten-Sherman transformation $\zeta_{\bmod A}$ is not surjective for the ring $A=\mathbb{Z} C_{2}$; see [17, prop. 5.1].

(8.4) Proposition. If the Auslander-Reiten homomorphism from Definition (2.3) is injective, then the following assertions hold:

(a) The homomorphism $\zeta_{\bmod R}: \mathrm{K}_{1}^{\mathrm{B}}(\bmod R) \rightarrow \mathrm{K}_{1}(\bmod R)$ is an isomorphism.

(b) There is an exact sequence,

$$
\mathrm{K}_{1}^{\mathrm{B}}(\mathcal{Y}) \stackrel{\mathrm{K}_{1}^{\mathrm{B}}(i)}{\longrightarrow} \mathrm{K}_{1}^{\mathrm{B}}(\bmod (\mathrm{MCM} R)) \stackrel{\mathrm{K}_{1}^{\mathrm{B}}(r)}{\longrightarrow} \mathrm{K}_{1}^{\mathrm{B}}(\bmod (\operatorname{proj} R)) \longrightarrow 0 .
$$

Proof. The Gabriel localization sequence (7.0.1) induces by (4.5) a long exact sequence of Quillen K-groups,

$$
\cdots \longrightarrow \mathrm{K}_{1}(\mathcal{Y}) \stackrel{\mathrm{K}_{1}(i)}{\longrightarrow} \mathrm{K}_{1}(\bmod (\mathrm{MCM} R)) \stackrel{\mathrm{K}_{1}(r)}{\longrightarrow} \mathrm{K}_{1}(\bmod (\operatorname{proj} R)) \longrightarrow \mathrm{K}_{0}(\mathcal{Y}) \stackrel{\mathrm{K}_{0}(i)}{\longrightarrow} \cdots
$$

By Proposition (7.3), we may identify $\mathrm{K}_{0}(i)$ with the Auslander-Reiten homomorphism, which is assumed to be injective. Therefore, the bottom row in the following 
commutative diagram of abelian groups is exact,

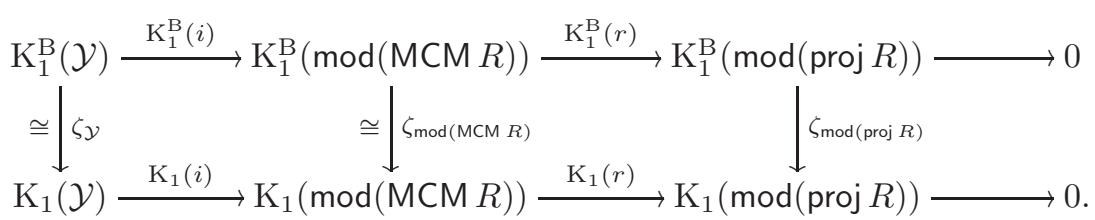

The vertical homomorphisms are given by the Gersten-Sherman transformation; see Section 3. It follows from Theorems (7.1) and (7.2) that $\mathcal{Y}$ is a length category with only finitely many simple objects; thus $\zeta_{\mathcal{Y}}$ is an isomorphism by Theorem (3.8). And $\zeta_{\bmod (\mathrm{MCM} R)}$ is an isomorphism by Lemma (8.3). Since $r i=0$, it follows that $\mathrm{K}_{1}^{\mathrm{B}}(r) \mathrm{K}_{1}^{\mathrm{B}}(i)=0$ holds, and a diagram chase now shows that $\operatorname{Im~}_{1}^{\mathrm{B}}(i)=\operatorname{Ker}_{1}^{\mathrm{B}}(r)$. Furthermore $\mathrm{K}_{1}^{\mathrm{B}}(r)$ is surjective by Lemma (8.2). This proves part (b).

The Five Lemma now implies that $\zeta_{\bmod (\operatorname{proj} R)}$ is an isomorphism. Since the category $\bmod (\operatorname{proj} R)$ is equivalent to $\bmod R$, see Observation (6.3), it follows that $\zeta_{\bmod R}$ is an isomorphism as well. This proves (a).

We will also need the following classical notion.

(8.5) Definition. Let $\mathcal{M}$ be an abelian category, and let $M$ be an object in $\mathcal{M}$. A projective cover of $M$ is an epimorphism $\varepsilon: P \rightarrow M$ in $\mathcal{M}$, where $P$ is projective, such that every endomorphism $\alpha: P \rightarrow P$ satisfying $\varepsilon \alpha=\varepsilon$ is an automorphism.

(8.6) Lemma. Let there be given a commutative diagram,

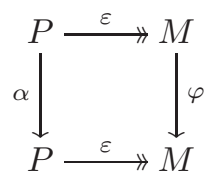

in an abelian category $\mathcal{M}$, where $\varepsilon: P \rightarrow M$ is a projective cover of $M$. If $\varphi$ is an automorphism, then $\alpha$ is an automorphism.

Proof. As $P$ is projective and $\varepsilon$ is an epimorphism, there exists $\beta: P \rightarrow P$ such that $\varepsilon \beta=\varphi^{-1} \varepsilon$. By assumption one has $\varepsilon \alpha=\varphi \varepsilon$. Hence $\varepsilon \alpha \beta=\varphi \varepsilon \beta=\varphi \varphi^{-1} \varepsilon=\varepsilon$, and similarly, $\varepsilon \beta \alpha=\varepsilon$. As $\varepsilon$ is a projective cover, we conclude that $\alpha \beta$ and $\beta \alpha$ are automorphisms of $P$, and thus $\alpha$ must be an automorphism.

The following lemma explains the point of the tilde construction (2.6).

(8.7) Lemma. Consider the isomorphism $\eta_{E^{\mathrm{op}}}: \mathrm{K}_{1}^{\mathrm{C}}\left(E^{\mathrm{op}}\right) \rightarrow \mathrm{K}_{1}^{\mathrm{B}}\left(\operatorname{proj}\left(E^{\mathrm{op}}\right)\right)$ in (3.5)). Let $X \in \operatorname{MCM} R$ and $\alpha \in \operatorname{Aut}_{R}(X)$ be given, and $\tilde{\alpha}$ be the invertible matrix with entries in $E$ obtained by applying Construction (2.6) to $\alpha$. There is an equality,

$$
\eta_{E^{\mathrm{op}}}\left(\tilde{\alpha}^{T}\right)=\left[\operatorname{Hom}_{R}(M, X), \operatorname{Hom}_{R}(M, \alpha)\right] .
$$

Proof. Write $(M,-)$ for $\operatorname{Hom}_{R}(M,-)$, and let $\psi: X \oplus Y \stackrel{\cong}{\longrightarrow} M^{q}$ be as in Construction (2.6). The $R$-module isomorphism $\psi$ induces an isomorphism of $E^{\mathrm{op}}$-modules,

$$
(M, X) \oplus(M, Y)=(M, X \oplus Y) \stackrel{(M, \psi)}{\cong}\left(M, M^{q}\right) \cong E^{q} .
$$

Consider the automorphism of the free $E^{\mathrm{op}}$-module $E^{q}$ given by

$$
(M, \psi)\left((M, \alpha) \oplus 1_{(M, Y)}\right)(M, \psi)^{-1}=\left(M, \psi\left(\alpha \oplus 1_{Y}\right) \psi^{-1}\right)=(M, \tilde{\alpha}) .
$$


We view elements in the $R$-module $M^{q}$ as columns and elements in $E^{q}$ as rows. The isomorphism $E^{q} \cong\left(M, M^{q}\right)$ identifies a row vector $\beta=\left(\beta_{1}, \ldots, \beta_{q}\right) \in E^{q}$ with the $R$-linear map $\beta^{T}: M \rightarrow M^{q}$ whose coordinate functions are $\beta_{1}, \ldots, \beta_{q}$. The coordinate functions of $(M, \tilde{\alpha})\left(\beta^{T}\right)=\tilde{\alpha} \circ \beta^{T}$ are the entries in the column $\tilde{\alpha} \beta^{T}$, where the matrix product used is $\mathrm{M}_{q \times q}(E) \times \mathrm{M}_{q \times 1}(E) \rightarrow \mathrm{M}_{q \times 1}(E)$. Thus, the action of $(M, \tilde{\alpha})$ on a row $\beta \in E^{q}$ is the row $\left(\tilde{\alpha} \beta^{T}\right)^{T} \in E^{q}$. In view of Remark (5.6) one has $\left(\tilde{\alpha} \beta^{T}\right)^{T}=\beta \cdot \tilde{\alpha}^{T}$, where "." is the product $\mathrm{M}_{1 \times q}\left(E^{\mathrm{op}}\right) \times \mathrm{M}_{q \times q}\left(E^{\mathrm{op}}\right) \rightarrow \mathrm{M}_{1 \times q}\left(E^{\mathrm{op}}\right)$. Consequently, over the ring $E^{\mathrm{op}}$, the automorphism $(M, \tilde{\alpha})$ of the $E^{\mathrm{op}}$-module $E^{q}$ acts on row vectors by multiplication with $\tilde{\alpha}^{T}$ from the right. These arguments show that $\eta_{E^{\text {op }}}^{-1}$ applied to $[(M, X),(M, \alpha)]$ is $\tilde{\alpha}^{T}$; see (3.5).

(8.8) Proposition. Suppose, in addition to the blanket assumptions for this section, that $R$ is an algebra over its residue field $k$ and that $\operatorname{char}(k) \neq 2$. Then there is a group isomorphism,

$$
\sigma: \operatorname{Aut}_{R}(M)_{\mathrm{ab}} \stackrel{\cong}{\longrightarrow} \mathrm{K}_{1}^{\mathrm{B}}(\bmod (\mathrm{MCM} R)),
$$

given by

$$
\alpha \longmapsto\left[\left.\operatorname{Hom}_{R}(-, M)\right|_{\mathrm{MCM} R},\left.\operatorname{Hom}_{R}(-, \alpha)\right|_{\mathrm{MCM} R}\right] .
$$

Furthermore, there is an equality,

$$
\sigma(\Xi)=\operatorname{Im}_{1}^{\mathrm{B}}(i) .
$$

Here $\Xi$ is the subgroup of $\operatorname{Aut}_{R}(M)_{\text {ab }}$ given in (2.10), and $i: \mathcal{Y} \rightarrow \bmod (\mathrm{MCM} R)$ is the inclusion functor from the Gabriel localization sequence (7.0.1).

Proof. We define $\sigma$ to be the composite of the following isomorphisms,

$$
\begin{aligned}
& \operatorname{Aut}_{R}(M)_{\mathrm{ab}}=E_{\mathrm{ab}}^{*}=\left(E^{\mathrm{op}}\right)_{\mathrm{ab}}^{*} \stackrel{\theta_{E^{\mathrm{op}}}}{\cong} \mathrm{K}_{1}^{\mathrm{C}}\left(E^{\mathrm{op}}\right) \\
& \stackrel{\eta_{E^{\mathrm{op}}}}{\cong} \mathrm{K}_{1}^{\mathrm{B}}\left(\operatorname{proj}\left(E^{\mathrm{op}}\right)\right) \\
& \stackrel{\mathrm{K}_{1}^{\mathrm{B}}(\jmath)}{\cong} \mathrm{K}_{1}^{\mathrm{B}}\left(\bmod \left(E^{\mathrm{op}}\right)\right) \\
& \stackrel{\mathrm{K}_{1}^{\mathrm{B}}\left(f_{M}\right)}{\cong} \mathrm{K}_{1}^{\mathrm{B}}(\bmod (\mathrm{MCM} R)) .
\end{aligned}
$$

The ring $E$, and hence also its opposite ring $E^{\mathrm{op}}$, is semilocal by Lemma (5.1). By assumption, $R$ is a $k$-algebra, and hence so is $E^{\mathrm{op}}$. Thus, in view of Remark (5.4) and the assumption $\operatorname{char}(k) \neq 2$, we get the isomorphism $\theta_{E^{\text {op }}}$ from Theorem (5.3). It maps $\alpha \in \operatorname{Aut}_{R}(M)_{\text {ab }}$ to the image of the $1 \times 1$ matrix $(\alpha) \in \mathrm{GL}\left(E^{\mathrm{op}}\right)$ in $\mathrm{K}_{1}^{\mathrm{C}}\left(E^{\mathrm{op}}\right)$.

The isomorphism $\eta_{E^{\mathrm{op}}}$ is described in (3.5); it maps $\xi \in \mathrm{GL}_{n}\left(E^{\mathrm{op}}\right)$ to the class $\left[\left(E_{E}\right)^{n}, \xi\right] \in \mathrm{K}_{1}^{\mathrm{B}}\left(\operatorname{proj}\left(E^{\mathrm{op}}\right)\right)$.

The third map in (8.8.1) is induced by the inclusion $\jmath: \operatorname{proj}\left(E^{\mathrm{op}}\right) \rightarrow \bmod \left(E^{\mathrm{op}}\right)$. By Leuschke [21, thm. 6] the noetherian ring $E^{\text {op }}$ has finite global dimension and hence Bass' resolution theorem [7, VIII§4 thm. (4.6)], or Rosenberg [25] thm. 3.1.14], implies that $\mathrm{K}_{1}^{\mathrm{B}}(\jmath)$ is an isomorphism. It maps an element $[P, \alpha] \in \mathrm{K}_{1}^{\mathrm{B}}\left(\operatorname{proj}\left(E^{\mathrm{op}}\right)\right)$ to $[P, \alpha] \in \mathrm{K}_{1}^{\mathrm{B}}\left(\bmod \left(E^{\mathrm{op}}\right)\right)$.

The fourth and last isomorphism $\mathrm{K}_{1}^{\mathrm{B}}\left(f_{M}\right)$ in (8.8.1) is induced by the equivalence $f_{M}: \bmod \left(E^{\mathrm{op}}\right) \rightarrow \bmod (\mathrm{MCM} R)$ from Proposition (6.2).

Thus, $\sigma$ is an isomorphism that maps an element $\alpha \in \operatorname{Aut}_{R}(M)_{\text {ab }}$ to the class

$$
\left[\left.E_{E} \otimes_{E} \operatorname{Hom}_{R}(-, M)\right|_{\text {MCM } R},\left.(\alpha \cdot) \otimes_{E} \operatorname{Hom}_{R}(-, M)\right|_{\text {MCM } R}\right],
$$


which is evidently the same as the class

$$
\left[\left.\operatorname{Hom}_{R}(-, M)\right|_{\text {MCM } R},\left.\operatorname{Hom}_{R}(-, \alpha)\right|_{\text {MCM } R}\right] \text {. }
$$

It remains to show the equality $\sigma(\Xi)=\operatorname{Im~K}_{1}^{\mathrm{B}}(i)$. By the definition 8.8.1) of $\sigma$ this is tantamount to showing that $\mathrm{K}_{1}^{\mathrm{B}}(\jmath) \eta_{E^{\mathrm{op}}} \theta_{E^{\text {op }}}(\Xi)=\mathrm{K}_{1}^{\mathrm{B}}\left(f_{M}\right)^{-1}\left(\operatorname{Im} \mathrm{K}_{1}^{\mathrm{B}}(i)\right)$. As $e_{M}$ is a quasi-inverse of $f_{M}$, see Proposition (6.2), we have $\mathrm{K}_{1}^{\mathrm{B}}\left(f_{M}\right)^{-1}=\mathrm{K}_{1}^{\mathrm{B}}\left(e_{M}\right)$, and hence we need to show the equality

$$
\mathrm{K}_{1}^{\mathrm{B}}(\jmath) \eta_{E^{\mathrm{op}}} \theta_{E^{\mathrm{op}}}(\Xi)=\mathrm{K}_{1}^{\mathrm{B}}\left(e_{M}\right)\left(\operatorname{Im~K}_{1}^{\mathrm{B}}(i)\right) .
$$

By Definition (2.10), the group $\Xi$ is generated by all elements of the form

$$
\xi_{j, \alpha}:=\left(\operatorname{det}_{E} \tilde{\alpha}\right)\left(\operatorname{det}_{E} \tilde{\beta}_{j, \alpha}\right)^{-1}\left(\operatorname{det}_{E} \tilde{\gamma}_{j, \alpha}\right) \in E_{\mathrm{ab}}^{*}
$$

for $j \in\{1, \ldots, t\}$ and $\alpha \in \operatorname{Aut}_{R}\left(M_{j}\right)$; here $\beta_{j, \alpha} \in \operatorname{Aut}_{R}\left(X_{j}\right)$ and $\gamma_{j, \alpha} \in \operatorname{Aut}_{R}\left(\tau\left(M_{j}\right)\right)$ are choices of automorphisms such that the diagram (2.9.1) is commutative. It follows from Lemma (5.7) that

$$
\xi_{j, \alpha}=\left(\operatorname{det}_{E^{\mathrm{op}}} \tilde{\alpha}^{T}\right)\left(\operatorname{det}_{E^{\mathrm{op}}} \tilde{\beta}_{j, \alpha}^{T}\right)^{-1}\left(\operatorname{det}_{E^{\mathrm{op}}} \tilde{\gamma}_{j, \alpha}^{T}\right) \in\left(E^{\mathrm{op}}\right)_{\mathrm{ab}}^{*} .
$$

By Definition (5.5) the homomorphism $\operatorname{det}_{E^{\mathrm{op}}}$ is the inverse of $\theta_{E^{\mathrm{op}}}$, and consequently the group $\theta_{E^{\mathrm{op}}}(\Xi)$ is generated by the elements

$$
\xi_{j, \alpha}^{\prime}:=\theta_{E_{\mathrm{op}}}\left(\xi_{j, \alpha}\right)=\tilde{\alpha}^{T}\left(\tilde{\beta}_{j, \alpha}^{T}\right)^{-1} \tilde{\gamma}_{j, \alpha}^{T} \in \mathrm{K}_{1}^{\mathrm{C}}\left(E^{\mathrm{op}}\right) .
$$

Thus $\eta_{E_{\mathrm{op}}} \theta_{E^{\mathrm{op}}}(\Xi)$ is generated by the elements $\xi_{j, \alpha}^{\prime \prime}:=\eta_{E_{\mathrm{op}}}\left(\xi_{j, \alpha}^{\prime}\right) \in \mathrm{K}_{1}^{\mathrm{B}}\left(\operatorname{proj}\left(E^{\mathrm{op}}\right)\right)$, and it follows from Lemma (8.7) that

$$
\begin{aligned}
\xi_{j, \alpha}^{\prime \prime}=[ & \left.\operatorname{Hom}_{R}\left(M, M_{j}\right), \operatorname{Hom}_{R}(M, \alpha)\right]-\left[\operatorname{Hom}_{R}\left(M, X_{j}\right), \operatorname{Hom}_{R}\left(M, \beta_{j, \alpha}\right)\right] \\
& +\left[\operatorname{Hom}_{R}\left(M, \tau\left(M_{j}\right)\right), \operatorname{Hom}_{R}\left(M, \gamma_{j, \alpha}\right)\right] .
\end{aligned}
$$

Thus, the group $\mathrm{K}_{1}^{\mathrm{B}}(\jmath) \eta_{E^{\mathrm{op}}} \theta_{E^{\mathrm{op}}}(\Xi)$ on the left-hand side in $(8.8 .2)$ is generated by the elements $\mathrm{K}_{1}^{\mathrm{B}}(\jmath)\left(\xi_{j, \alpha}^{\prime \prime}\right)$. Note that $\mathrm{K}_{1}^{\mathrm{B}}(\jmath)\left(\xi_{j, \alpha}^{\prime \prime}\right)$ is nothing but $\xi_{j, \alpha}^{\prime \prime}$ viewed as an element in $\mathrm{K}_{1}^{\mathrm{B}}\left(\bmod \left(E^{\mathrm{op}}\right)\right)$. We have reached the following conclusion:

The group $\mathrm{K}_{1}^{\mathrm{B}}(\jmath) \eta_{E^{\mathrm{op}}} \theta_{E^{\mathrm{op}}}(\Xi)$ is generated by the elements $\xi_{j, \alpha}^{\prime \prime}$, where $j$ ranges over $\{1, \ldots, t\}$ and $\alpha$ over all automorphisms of $M_{j}$.

To give a useful set of generators of the group $\mathrm{K}_{1}^{\mathrm{B}}\left(e_{M}\right)\left(\operatorname{Im~K}_{1}^{\mathrm{B}}(i)\right)$ on the righthand side in (8.8.2), recall from Theorems (7.1) and (7.2) that every element in $\mathcal{Y}$ has finite length and that the simple objects in $\mathcal{Y}$ are, up to isomorphism, exactly the functors $F_{1}, \ldots, F_{t}$. Thus, by [25, (proof of) thm. 3.1.8(2)] the group $\mathrm{K}_{1}^{\mathrm{B}}(\mathcal{Y})$ is generated by all elements of the form $\left[F_{j}, \varphi\right]$, where $j \in\{1, \ldots, t\}$ and $\varphi$ is an automorphism of $F_{j}$. It follows that the group $\operatorname{Im~K}_{1}^{\mathrm{B}}(i)$ is generated by the elements $\mathrm{K}_{1}^{\mathrm{B}}(i)\left(\left[F_{j}, \varphi\right]\right)$. Note that $\mathrm{K}_{1}^{\mathrm{B}}(i)\left(\left[F_{j}, \varphi\right]\right)$ is nothing but $\left[F_{j}, \varphi\right]$ viewed as an element in $\mathrm{K}_{1}^{\mathrm{B}}(\bmod (\mathrm{MCM} R))$. By definition of the functor $e_{M}$, see Prop. (6.2), one has

$$
\lambda_{j, \varphi}:=\mathrm{K}_{1}^{\mathrm{B}}\left(e_{M}\right)\left(\left[F_{j}, \varphi\right]\right)=\left[F_{j} M, \varphi_{M}\right] .
$$

We have reached the following conclusion:

The group $\mathrm{K}_{1}^{\mathrm{B}}\left(e_{M}\right)\left(\operatorname{Im~K}_{1}^{\mathrm{B}}(i)\right)$ is generated by the elements $\lambda_{j, \varphi}$, where $j$ ranges over $\{1, \ldots, t\}$ and $\varphi$ over all automorphisms of $F_{j}$.

With the descriptions of the generators $\xi_{j, \alpha}^{\prime \prime}$ and $\lambda_{j, \varphi}$ at hand, we are now in a position to prove the identity (8.8.2). 
Consider an arbitrary generator $\xi_{j, \alpha}^{\prime \prime}$ in the group $K_{1}^{\mathrm{B}}(\jmath) \eta_{E^{\mathrm{op}}} \theta_{E^{\mathrm{op}}}(\Xi)$. Recall from Theorem (7.2) that there is an exact sequence in $\bmod (\operatorname{MCM} R)$,

$$
0 \longrightarrow \operatorname{Hom}_{R}\left(-, \tau\left(M_{j}\right)\right) \longrightarrow \operatorname{Hom}_{R}\left(-, X_{j}\right) \longrightarrow \operatorname{Hom}_{R}\left(-, M_{j}\right) \longrightarrow F_{j} \longrightarrow 0 \text {. }
$$

Thus, the commutative diagram 2.9.1) in MCM $R$ induces a commutative diagram in $\bmod (\mathrm{MCM} R)$ with exact $\operatorname{row}(\mathrm{s})$,

$$
\begin{aligned}
& 0 \longrightarrow \operatorname{Hom}_{R}\left(-, \tau\left(M_{j}\right)\right) \longrightarrow \operatorname{Hom}_{R}\left(-, X_{j}\right) \longrightarrow \operatorname{Hom}_{R}\left(-, M_{j}\right) \longrightarrow F_{j} \longrightarrow 0
\end{aligned}
$$

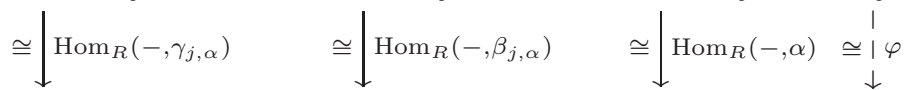

$$
\begin{aligned}
& 0 \longrightarrow \operatorname{Hom}_{R}\left(-, \tau\left(M_{j}\right)\right) \longrightarrow \operatorname{Hom}_{R}\left(-, X_{j}\right) \longrightarrow \operatorname{Hom}_{R}\left(-, M_{j}\right) \longrightarrow \stackrel{\downarrow}{F_{j}} \longrightarrow 0 \text {, }
\end{aligned}
$$

where $\varphi$ is the uniquely determined natural endotransformation of $F_{j}$ that makes this diagram commutative. Note that $\varphi$ is an automorphism by the Five Lemma, and thus $\left[F_{j}, \varphi\right]$ is a well-defined element in $\mathrm{K}_{1}^{\mathrm{B}}(\bmod (\operatorname{MCM} R))$. The diagram above is an exact sequence in the loop category $\Omega(\bmod (\mathrm{MCM} R))$, see (3.2) and (3.3), so in the group $\mathrm{K}_{1}^{\mathrm{B}}(\bmod (\mathrm{MCM} R))$ there is an equality:

$$
\begin{aligned}
{\left[F_{j}, \varphi\right]=[} & \left.\operatorname{Hom}_{R}\left(-, M_{j}\right), \operatorname{Hom}_{R}(-, \alpha)\right]-\left[\operatorname{Hom}_{R}\left(-, X_{j}\right), \operatorname{Hom}_{R}\left(-, \beta_{j, \alpha}\right)\right] \\
& +\left[\operatorname{Hom}_{R}\left(-, \tau\left(M_{j}\right)\right), \operatorname{Hom}_{R}\left(-, \gamma_{j, \alpha}\right)\right] .
\end{aligned}
$$

Applying the homomorphism $\mathrm{K}_{1}^{\mathrm{B}}\left(e_{M}\right)$ to this equality, we get $\lambda_{j, \varphi}=\xi_{j, \alpha}^{\prime \prime}$. These arguments show that every generator $\xi_{j, \alpha}^{\prime \prime}$ has the form $\lambda_{j, \varphi}$ for some $\varphi$, and hence the inclusion " $\subseteq$ " in (8.8.2) is established.

Conversely, consider an arbitrary generator $\lambda_{j, \varphi}$ in the group $\mathrm{K}_{1}^{\mathrm{B}}\left(e_{M}\right)\left(\operatorname{Im~K}_{1}^{\mathrm{B}}(i)\right)$. As the category MCM $R$ is a Krull-Schmidt variety in the sense of Auslander [1, II, $\S 2]$, it follows by [1, II, prop. 2.1(b,c)] and [1, I, prop. 4.7] that $\operatorname{Hom}_{R}\left(-, M_{j}\right) \rightarrow F_{j}$ is a projective cover in $\bmod (\mathrm{MCM} R)$ in the sense of Definition (8.5). In particular, $\varphi$ lifts to a natural transformation $\psi$ of $\operatorname{Hom}_{R}\left(-, M_{j}\right)$, which must be an automorphism by Lemma (8.6). Thus we have a commutative diagram in $\bmod (\mathrm{MCM} R)$,

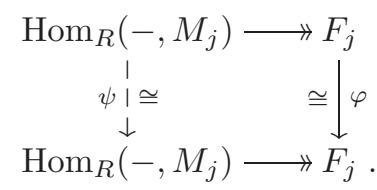

As the Yoneda functor $y_{M}: \operatorname{MCM} R \rightarrow \bmod (\operatorname{MCM} R)$ is fully faithful, see [32, lem. (4.3)], there exists a unique automorphism $\alpha$ of $M_{j}$ such that $\psi=\operatorname{Hom}_{R}(-, \alpha)$. For this particular $\alpha$, the arguments above show that $\lambda_{j, \varphi}=\xi_{j, \alpha}^{\prime \prime}$. Thus every generator $\lambda_{j, \varphi}$ has the form $\xi_{j, \alpha}^{\prime \prime}$ for some $\alpha$, and hence the inclusion " $\supseteq$ " in (8.8.2) holds.

(8.9) Observation. For any commutative noetherian local ring $R$, there is an isomorphism $\rho_{R}: R^{*} \stackrel{\cong}{\longrightarrow} \mathrm{K}_{1}^{\mathrm{B}}($ proj $R$ ) given by the composite of

$$
R^{*} \stackrel{\theta_{R}}{\cong} \mathrm{K}_{1}^{\mathrm{C}}(R) \stackrel{\eta_{R}}{\cong} \mathrm{K}_{1}^{\mathrm{B}}(\operatorname{proj} R)
$$

The first map is described in (5.2); it is an isomorphism by Srinivas [27, exa. (1.6)]. The second isomorphism is discussed in (3.5). Thus, $\rho_{R}$ maps $r \in R^{*}$ to $\left[R, r 1_{R}\right]$.

We are finally in a position to prove the main result.

Proof of Theorem (2.12). By Proposition (8.4) we can identify $\mathrm{K}_{1}(\bmod R)$ with the group $\mathrm{K}_{1}^{\mathrm{B}}(\bmod R)$. Recall that $i$ and $r$ denote the inclusion and restriction functors from the localization sequence (7.0.1). By the relations that define $\mathrm{K}_{1}^{\mathrm{B}}(\bmod R)$, see 
(3.3), there is a homomorphism $\pi_{0}: \operatorname{Aut}_{R}(M) \rightarrow \mathrm{K}_{1}^{\mathrm{B}}(\bmod R)$ given by $\alpha \mapsto[M, \alpha]$. Since $\mathrm{K}_{1}^{\mathrm{B}}(\bmod R)$ is abelian, $\pi_{0}$ induces a homomorphism $\pi$, which is displayed as the upper horizontal map in the following diagram,

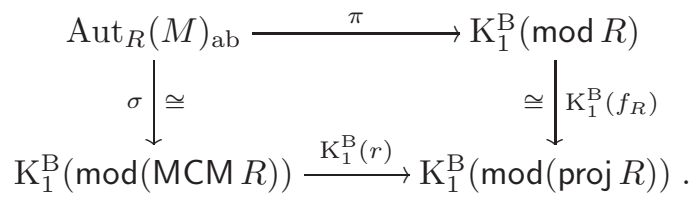

Here $\sigma$ is the isomorphism from Proposition (8.8), and the isomorphism $\mathrm{K}_{1}^{\mathrm{B}}\left(f_{R}\right)$ is induced by the equivalence $f_{R}$ from Observation (6.3). The diagram (8.9.1) is commutative, indeed, $\mathrm{K}_{1}^{\mathrm{B}}(r) \sigma$ and $\mathrm{K}_{1}^{\mathrm{B}}\left(f_{R}\right) \pi$ both map $\alpha \in \operatorname{Aut}_{R}(M)$ ab to the class

$$
\left[\left.\operatorname{Hom}_{R}(-, M)\right|_{\operatorname{proj} R},\left.\operatorname{Hom}_{R}(-, \alpha)\right|_{\text {proj } R}\right] \text {. }
$$

By Lemma (8.2) the homomorphism $\mathrm{K}_{1}^{\mathrm{B}}(r)$ is surjective, and hence so is $\pi$. Exactness of the sequence in Proposition (8.4) (b) and commutativity of the diagram (8.9.1) show that $\operatorname{Ker} \pi=\sigma^{-1}\left(\operatorname{Im~K}_{1}^{\mathrm{B}}(i)\right)$. Therefore Proposition (8.8) implies that there is an equality $\operatorname{Ker} \pi=\Xi$, and it follows that $\pi$ induces an isomorphism,

$$
\widehat{\pi}: \operatorname{Aut}_{R}(M)_{\mathrm{ab}} / \Xi \stackrel{\cong}{\longrightarrow} \mathrm{K}_{1}^{\mathrm{B}}(\bmod R) .
$$

This proves the first assertion in Theorem (2.12).

To prove the second assertion, let inc: $\operatorname{proj} R \rightarrow \bmod R$ denote the inclusion functor. Note that the Gersten-Sherman transformation identifies the homomorphisms $\mathrm{K}_{1}$ (inc) and $\mathrm{K}_{1}^{\mathrm{B}}$ (inc); indeed $\zeta_{\text {proj } R}$ is an isomorphism by Theorem (3.7) and $\zeta_{\bmod R}$ is an isomorphism by Proposition (8.4) (a). Thus, we must show that $\mathrm{K}_{1}^{\mathrm{B}}$ (inc) can be identified with the homomorphism $\lambda: R^{*} \rightarrow \operatorname{Aut}_{R}(M)_{\text {ab }} / \Xi$ given by $r \mapsto r 1_{R} \oplus 1_{M^{\prime}}$ (recall that we have written $M=R \oplus M^{\prime}$ ). To this end, consider the isomorphism $\rho_{R}: R^{*} \rightarrow \mathrm{K}_{1}^{\mathrm{B}}(\operatorname{proj} R)$ from Observation (8.9) given by $r \mapsto\left[R, r 1_{R}\right]$. The fact that $\mathrm{K}_{1}^{\mathrm{B}}$ (inc) and $\lambda$ are isomorphic maps now follows from the diagram,

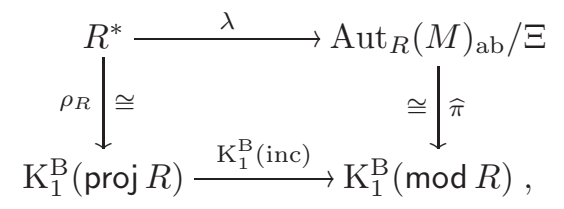

which is commutative. Indeed, for $r \in R^{*}$ one has

$$
(\widehat{\pi} \lambda)(r)=\left[M, r 1_{R} \oplus 1_{M^{\prime}}\right]=\left[R, r 1_{R}\right]+\left[M^{\prime}, 1_{M^{\prime}}\right]=\left[R, r 1_{R}\right]=\left(\mathrm{K}_{1}^{\mathrm{B}}(\mathrm{inc}) \rho_{R}\right)(r),
$$

where the penultimate equality is by (3.4).

\section{Abelianization of Automorphism Groups}

To apply Theorem (2.12), one must compute $\operatorname{Aut}_{R}(M)_{\mathrm{ab}}$, i.e. the abelianization of the automorphism group of the representation generator $M$. In Proposition (9.6) we compute $\operatorname{Aut}_{R}(M)_{\text {ab }}$ for the $R$-module $M=R \oplus \mathfrak{m}$, which is a representation generator for MCM $R$ if $\mathfrak{m}$ happens to be the only non-free indecomposable maximal Cohen-Macaulay module over $R$. Specific examples of rings for which this is the case will be studied in Section 10. Throughout this section, $A$ denotes any ring. 
(9.1) Definition. Let $N_{1}, \ldots, N_{s}$ be $A$-modules, and set $N=N_{1} \oplus \cdots \oplus N_{s}$. We view elements in $N$ as column vectors.

For $\varphi \in \operatorname{Aut}_{A}\left(N_{i}\right)$ we denote by $d_{i}(\varphi)$ the automorphism of $N$ which has as its diagonal $1_{N_{1}}, \ldots, 1_{N_{i-1}}, \varphi, 1_{N_{i+1}}, \ldots, 1_{N_{s}}$ and 0 in all other entries.

For $i \neq j$ and $\mu \in \operatorname{Hom}_{A}\left(N_{j}, N_{i}\right)$ we denote by $e_{i j}(\mu)$ the automorphism of $N$ with diagonal $1_{N_{1}}, \ldots, 1_{N_{s}}$, and whose only non-trivial off-diagonal entry is $\mu$ in position $(i, j)$.

(9.2) Lemma. Let $N_{1}, \ldots, N_{s}$ be $A$-modules and set $N=N_{1} \oplus \cdots \oplus N_{s}$. If $2 \in A$ is a unit, if $i \neq j$, and if $\mu \in \operatorname{Hom}_{A}\left(N_{j}, N_{i}\right)$ then $e_{i j}(\mu)$ is a commutator in $\operatorname{Aut}_{A}(N)$.

Proof. The commutator of $\varphi$ and $\psi$ in $\operatorname{Aut}_{A}(N)$ is $[\varphi, \psi]=\varphi \psi \varphi^{-1} \psi^{-1}$. It is easily verified that $e_{i j}(\mu)=\left[e_{i j}\left(\frac{\mu}{2}\right), d_{j}\left(-1_{N_{j}}\right)\right]$ if $i \neq j$.

The idea in the proof above is certainly not new. It appears, for example, already in Litoff [22, proof of thm. 2] in the case $s=2$. Of course, if $s \geqslant 3$ then $e_{i j}(\mu)$ is a commutator even without the assumption that 2 is a unit; see e.g. 225, lem. 2.1.2(c)].

(9.3) Lemma. Let $X$ and $Y$ be non-isomorphic A-modules with local endomorphism rings. Let $\varphi, \psi \in \operatorname{End}_{A}(X)$ and assume that $\psi$ factors through $Y$. Then one has $\psi \notin \operatorname{Aut}_{A}(X)$. Futhermore, $\varphi \in \operatorname{Aut}_{A}(X)$ if and only if $\varphi+\psi \in \operatorname{Aut}_{A}(X)$.

Proof. Write $\psi=\psi^{\prime \prime} \psi^{\prime}$ with $\psi^{\prime}: X \rightarrow Y$ and $\psi^{\prime \prime}: Y \rightarrow X$. If $\psi$ is an automorphism, then $\psi^{\prime \prime}$ is a split epimorphism and hence an isomorphism as $Y$ is indecomposable. This contradicts the assumption that $X$ and $Y$ are not isomorphic. The second assertion now follows as $\operatorname{Aut}_{A}(X)$ is the set of units in the local ring $\operatorname{End}_{A}(X)$.

(9.4) Proposition. Let $N_{1}, \ldots, N_{s}$ be pairwise non-isomorphic A-modules with local endomorphism rings. An endomorphism

$$
\alpha=\left(\alpha_{i j}\right) \in \operatorname{End}_{A}\left(N_{1} \oplus \cdots \oplus N_{s}\right) \quad \text { with } \quad \alpha_{i j} \in \operatorname{Hom}_{A}\left(N_{j}, N_{i}\right)
$$

is an automorphism if and only if $\alpha_{11}, \alpha_{22}, \ldots, \alpha_{s s}$ are automorphisms.

Furthermore, every $\alpha$ in $\operatorname{Aut}_{A}(N)$ can be written as a product of automorphisms of the form $d_{i}(\cdot)$ and $e_{i j}(\cdot)$, cf. Definition (9.1).

Proof. "Only if": Assume that $\alpha=\left(\alpha_{i j}\right)$ is an automorphism with inverse $\beta=\left(\beta_{i j}\right)$ and let $i=1, \ldots, s$ be given. In the local ring $\operatorname{End}_{A}\left(N_{i}\right)$ one has $1_{N_{i}}=\sum_{j=1}^{s} \alpha_{i j} \beta_{j i}$, and hence one of the terms $\alpha_{i j} \beta_{j i}$ must be an automorphism. As $\alpha_{i j} \beta_{j i}$ is not an automorphism for $j \neq i$, see Lemma (9.3), it follows that $\alpha_{i i} \beta_{i i}$ is an automorphism. In particular, $\alpha_{i i}$ has a right inverse and $\beta_{i i}$ has a left inverse, and since the ring $\operatorname{End}_{A}\left(N_{i}\right)$ is local this means that $\alpha_{i i}$ and $\beta_{i i}$ are both automorphisms.

"If": By induction on $s \geqslant 1$. The assertion is trivial for $s=1$. Now let $s>1$. Assume that $\alpha_{11}, \alpha_{22}, \ldots, \alpha_{s s}$ are automorphisms. Recall the notation from (9.1). By composing $\alpha$ with $e_{s 1}\left(-\alpha_{s 1} \alpha_{11}^{-1}\right) \cdots e_{31}\left(-\alpha_{31} \alpha_{11}^{-1}\right) e_{21}\left(-\alpha_{21} \alpha_{11}^{-1}\right)$ from the left and with $e_{12}\left(-\alpha_{11}^{-1} \alpha_{12}\right) e_{13}\left(-\alpha_{11}^{-1} \alpha_{13}\right) \cdots e_{1 s}\left(-\alpha_{11}^{-1} \alpha_{1 s}\right)$ from the right, one gets an endomorphism of the form

$$
\alpha^{\prime}=\left(\begin{array}{c|c}
\alpha_{11} & 0 \\
\hline 0 & \beta
\end{array}\right)=d_{1}\left(\alpha_{11}\right)\left(\begin{array}{c|c}
1_{N_{1}} & 0 \\
\hline 0 & \beta
\end{array}\right),
$$

where $\beta \in \operatorname{End}_{A}\left(N_{2} \oplus \cdots \oplus N_{s}\right)$ is an $(s-1) \times(s-1)$ matrix with diagonal entries given by $\alpha_{j j}-\alpha_{j 1} \alpha_{11}^{-1} \alpha_{1 j}$ for $j=2, \ldots, s$. By applying Lemma (9.3) to the situation $\varphi=\alpha_{j j}-\alpha_{j 1} \alpha_{11}^{-1} \alpha_{1 j}$ and $\psi=\alpha_{j 1} \alpha_{11}^{-1} \alpha_{1 j}$, it follows that the diagonal entries in 
$\beta$ are all automorphisms. By the induction hypothesis, $\beta$ is now an automorphism and can be written as a product of automorphisms of the form $d_{i}(\cdot)$ and $e_{i j}(\cdot)$. Consequently, the same is true for $\alpha^{\prime}$, and hence also for $\alpha$.

(9.5) Corollary. Assume that $2 \in A$ is a unit and let $N_{1}, \ldots, N_{s}$ be pairwise nonisomorphic A-modules with local endomorphism rings. The homomorphism,

$$
\Delta: \operatorname{Aut}_{A}\left(N_{1}\right) \times \cdots \times \operatorname{Aut}_{A}\left(N_{s}\right) \longrightarrow \operatorname{Aut}_{A}\left(N_{1} \oplus \cdots \oplus N_{s}\right),
$$

given by $\Delta\left(\varphi_{1}, \ldots, \varphi_{s}\right)=d_{1}\left(\varphi_{1}\right) \cdots d_{s}\left(\varphi_{s}\right)$, induces a surjective homomorphism,

$$
\Delta_{\mathrm{ab}}: \operatorname{Aut}_{A}\left(N_{1}\right)_{\mathrm{ab}} \oplus \cdots \oplus \operatorname{Aut}_{A}\left(N_{s}\right)_{\mathrm{ab}} \longrightarrow \operatorname{Aut}_{A}\left(N_{1} \oplus \cdots \oplus N_{s}\right)_{\mathrm{ab}} .
$$

Proof. By Proposition (9.4) every element in $\operatorname{Aut}_{A}\left(N_{1} \oplus \cdots \oplus N_{s}\right)$ is a product of automorphisms of the form $d_{i}(\cdot)$ and $e_{i j}(\cdot)$. As $2 \in A$ is a unit, Lemma (9.2) yields that every element of the form $e_{i j}(\cdot)$ is a commutator; thus in $\operatorname{Aut}_{A}\left(N_{1} \oplus \cdots \oplus N_{s}\right)_{\text {ab }}$ every element is a product of elements of the form $d_{i}(\cdot)$, so $\Delta_{\mathrm{ab}}$ is surjective.

As noted above, Lemma (9.2), and consequently also Corollary (9.5), holds without the assumption that $2 \in A$ is a unit provided that $s \geqslant 3$.

In the following, we write $[\cdot]_{\mathfrak{m}}: R \rightarrow R / \mathfrak{m}=k$ for the quotient homomorphism.

(9.6) Proposition. Let $(R, \mathfrak{m}, k)$ be any commutative local ring such that $2 \in R$ is a unit. Assume that $\mathfrak{m}$ is not isomorphic to $R$ and that the endomorphism ring $\operatorname{End}_{R}(\mathfrak{m})$ is commutative and local. There is an isomorphism of abelian groups,

$$
\delta: \operatorname{Aut}_{R}(R \oplus \mathfrak{m})_{\mathrm{ab}} \stackrel{\cong}{\longrightarrow} k^{*} \oplus \operatorname{Aut}_{R}(\mathfrak{m}) \text {, }
$$

given by

$$
\left(\begin{array}{ll}
\alpha_{11} & \alpha_{22} \\
\alpha_{21} & \alpha_{22}
\end{array}\right) \longmapsto\left(\left[\alpha_{11}(1)\right]_{\mathfrak{m}}, \alpha_{11} \alpha_{22}-\alpha_{21} \alpha_{12}\right) .
$$

Proof. First note that the image of any homomorphism $\alpha: \mathfrak{m} \rightarrow R$ is contained in $\mathfrak{m}$. Indeed if $\operatorname{Im} \alpha \nsubseteq \mathfrak{m}$, then $u=\alpha(a)$ is a unit for some $a \in \mathfrak{m}$, and thus $\alpha\left(u^{-1} a\right)=1$. It follows that $\alpha$ is surjective, and hence a split epimorphism as $R$ is free. Since $\mathfrak{m}$ is indecomposable, $\alpha$ must be an isomorphism, which is a contradiction.

Therefore, given an endomorphism,

$$
\left(\begin{array}{ll}
\alpha_{11} & \alpha_{12} \\
\alpha_{21} & \alpha_{22}
\end{array}\right) \in \operatorname{End}_{R}(R \oplus \mathfrak{m})=\left(\begin{array}{ll}
\operatorname{Hom}_{R}(R, R) & \operatorname{Hom}_{R}(\mathfrak{m}, R) \\
\operatorname{Hom}_{R}(R, \mathfrak{m}) & \operatorname{Hom}_{R}(\mathfrak{m}, \mathfrak{m})
\end{array}\right),
$$

we may by (co)restriction view the entries $\alpha_{i j}$ as elements in the endomorphism ring $\operatorname{End}_{R}(\mathfrak{m})$. As this ring is assumed to be commutative, the determinant map

$$
\operatorname{End}_{R}(R \oplus \mathfrak{m}) \longrightarrow \operatorname{End}_{R}(\mathfrak{m}) \quad \text { given by } \quad\left(\alpha_{i j}\right) \longmapsto \alpha_{11} \alpha_{22}-\alpha_{21} \alpha_{12}
$$

preserves multiplication. If $\left(\alpha_{i j}\right) \in \operatorname{Aut}_{R}(R \oplus \mathfrak{m})$, then Proposition (9.4) implies that $\alpha_{11} \in \operatorname{Aut}_{R}(R)$ and $\alpha_{22} \in \operatorname{Aut}_{R}(\mathfrak{m})$, and thus $\alpha_{11} \alpha_{22} \in \operatorname{Aut}_{R}(\mathfrak{m})$. By applying Lemma (9.3) to $\varphi=\alpha_{11} \alpha_{22}-\alpha_{21} \alpha_{12}$ and $\psi=\alpha_{21} \alpha_{12}$ we get $\varphi \in \operatorname{Aut}_{R}(\mathfrak{m})$, and hence the determinant map is a group homomorphism $\operatorname{Aut}_{R}(R \oplus \mathfrak{m}) \rightarrow \operatorname{Aut}_{R}(\mathfrak{m})$.

The map $\operatorname{Aut}_{R}(R \oplus \mathfrak{m}) \rightarrow k^{*}$ defined by $\left(\alpha_{i j}\right) \mapsto\left[\alpha_{11}(1)\right]_{\mathfrak{m}}$ is also a group homomorphism. Indeed, entry $(1,1)$ in the product $\left(\alpha_{i j}\right)\left(\beta_{i j}\right)$ is $\alpha_{11} \beta_{11}+\alpha_{12} \beta_{21}$. Here $\alpha_{12}$ is a homomorphism $\mathfrak{m} \rightarrow R$, and hence $\alpha_{12} \beta_{21}(1) \in \mathfrak{m}$ by the arguments in the beginning of the proof. Consequently one has

$$
\left[\left(\alpha_{11} \beta_{11}+\alpha_{12} \beta_{21}\right)(1)\right]_{\mathfrak{m}}=\left[\left(\alpha_{11} \beta_{11}\right)(1)\right]_{\mathfrak{m}}=\left[\alpha_{11}(1) \beta_{11}(1)\right]_{\mathfrak{m}}=\left[\alpha_{11}(1)\right]_{\mathfrak{m}}\left[\beta_{11}(1)\right]_{\mathfrak{m}} .
$$


These arguments and the fact that the groups $k^{*}$ and $\operatorname{Aut}_{R}(\mathfrak{m})$ are abelian show that the map $\delta$ described in the proposition is a well-defined group homomorphism. Evidently, $\delta$ is surjective; indeed, for $[r]_{\mathfrak{m}} \in k^{*}$ and $\varphi \in \operatorname{Aut}_{R}(\mathfrak{m})$ one has

$$
\delta\left(\begin{array}{cc}
r 1_{R} & 0 \\
0 & r^{-1} \varphi
\end{array}\right)=\left([r]_{\mathfrak{m}}, \varphi\right) .
$$

To show that $\delta$ is injective, assume that $\alpha \in \operatorname{Aut}_{R}(R \oplus \mathfrak{m})$ ab with $\delta(\alpha)=\left([1]_{\mathfrak{m}}, 1_{\mathfrak{m}}\right)$. By Corollary (9.5) we can assume that $\alpha=\left(\alpha_{i j}\right)$ is a diagonal matrix. We write $\alpha_{11}=r 1_{R}$ for some unit $r \in R$. Since one has $\delta(\alpha)=\left([r]_{\mathfrak{m}}, r \alpha_{22}\right)$ we conclude that $r \in 1+\mathfrak{m}$ and $\alpha_{22}=r^{-1} 1_{\mathfrak{m}}$, that is, $\alpha$ has the form

$$
\alpha=\left(\begin{array}{cc}
r 1_{R} & 0 \\
0 & r^{-1} 1_{\mathfrak{m}}
\end{array}\right) \quad \text { with } \quad r \in 1+\mathfrak{m} .
$$

Thus, proving injectivity of $\delta$ amounts to showing that every automorphism $\alpha$ of the form above belongs to the commutator subgroup of $\operatorname{Aut}_{R}(R \oplus \mathfrak{m})$. As $r-1 \in \mathfrak{m}$ the $\operatorname{map}(r-1) 1_{R}$ gives a homomorphism $R \rightarrow \mathfrak{m}$. Since $r\left(r^{-1}-1\right)=1-r \in \mathfrak{m}$ and $r \notin \mathfrak{m}$, it follows that $r^{-1}-1 \in \mathfrak{m}$. Thus $\left(r^{-1}-1\right) 1_{R}$ gives another homomorphism $R \rightarrow \mathfrak{m}$. If $\iota: \mathfrak{m} \hookrightarrow R$ denotes the inclusion, then one has 2

$$
\left(\begin{array}{cc}
r 1_{R} & 0 \\
0 & r^{-1} 1_{\mathfrak{m}}
\end{array}\right)=\left(\begin{array}{cc}
1_{R} & 0 \\
\left(r^{-1}-1\right) 1_{R} & 1_{\mathfrak{m}}
\end{array}\right)\left(\begin{array}{cc}
1_{R} & \iota \\
0 & 1_{\mathfrak{m}}
\end{array}\right)\left(\begin{array}{cc}
1_{R} & 0 \\
(r-1) 1_{R} & 1_{\mathfrak{m}}
\end{array}\right)\left(\begin{array}{cc}
1_{R} & -r^{-1} \iota \\
0 & 1_{\mathfrak{m}}
\end{array}\right) .
$$

The right-hand of this equality is a product of matrices of the form $e_{i j}(\cdot)$, and since $2 \in R$ is a unit the desired conclusion now follows from Lemma (9.2).

\section{EXAMPLES}

We begin with a trivial example.

(10.1) Example. If $R$ is regular, then there are isomorphisms,

$$
\mathrm{K}_{1}(\bmod R) \cong \mathrm{K}_{1}(\operatorname{proj} R) \cong \mathrm{K}_{1}^{\mathrm{C}}(R) \cong R^{*} .
$$

The first isomorphism is by Quillen's resolution theorem [24, $\S 4$ thm. 3], the second one is mentioned in (3.6), and the third one is well-known; see e.g. [27, exa. (1.6)]. Theorem (2.12) confirms this result, indeed, as $M=R$ is a representation generator for $\operatorname{MCM} R=\operatorname{proj} R$ one has $\operatorname{Aut}_{R}(M)_{\mathrm{ab}}=R^{*}$. As there are no Auslander-Reiten sequences in this case, the subgroup $\Xi$ is generated by the empty set, so $\Xi=0$.

We now illustrate how Theorem (2.12) applies to compute $\mathrm{K}_{1}(\bmod R)$ for the ring $R=k[X] /\left(X^{2}\right)$. The answer is well-known to be $k^{*}$, indeed, for any commutative artinian local ring $R$ with residue field $k$ one has $\mathrm{K}_{1}(\bmod R) \cong k^{*}$ by $[24, \S 5$ cor. 1$]$.

(10.2) Example. Let $R=k[X] /\left(X^{2}\right)$ be the ring of dual numbers over a field $k$ with $\operatorname{char}(k) \neq 2$. Denote by inc: $\operatorname{proj} R \rightarrow \bmod R$ the inclusion functor. The homomorphism $\mathrm{K}_{1}$ (inc) may be identified with the map,

$$
\mu: R^{*} \longrightarrow k^{*} \quad \text { given by } \quad a+b X \longmapsto a^{2} .
$$

Proof. The maximal ideal $\mathfrak{m}=(X)$ is the only non-free indecomposable maximal Cohen-Macaulay $R$-module, so $M=R \oplus \mathfrak{m}$ is a representation generator for MCM $R$; see (2.1.1). There is an isomorphism $k \rightarrow \operatorname{End}_{R}(\mathfrak{m})$ of $R$-algebras given by $a \mapsto a 1_{\mathfrak{m}}$,

\footnotetext{
2 The identity comes from the standard proof of Whitehead's lemma; see e.g. [27] lem. (1.4)].
} 
in particular, $\operatorname{End}_{R}(\mathfrak{m})$ is commutative. Via this isomorphism, $k^{*}$ corresponds to $\operatorname{Aut}_{R}(\mathfrak{m})$. The Auslander-Reiten sequence ending in $\mathfrak{m}$ is

$$
0 \longrightarrow \mathfrak{m} \stackrel{\iota}{\longrightarrow} R \stackrel{X}{\longrightarrow} \mathfrak{m} \longrightarrow 0,
$$

where $\iota$ is the inclusion. The Auslander-Reiten homomorphism $\Upsilon=\left(\begin{array}{c}-1 \\ 2\end{array}\right): \mathbb{Z} \rightarrow \mathbb{Z}^{2}$ is injective, so Theorem (2.12) can be applied. Note that for every $a 1_{\mathfrak{m}} \in \operatorname{Aut}_{R}(\mathfrak{m})$, where $a \in k^{*}$, there is a commutative diagram,

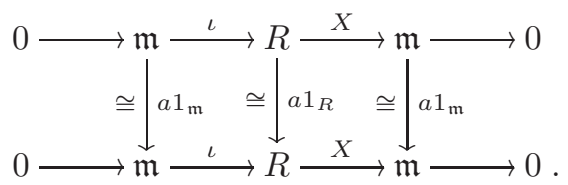

Applying the tilde construction (2.6) to the automorphisms $a 1_{\mathfrak{m}}$ and $a 1_{R}$ one gets

$$
\widetilde{a 1_{\mathfrak{m}}}=\left(\begin{array}{cc}
1_{R} & 0 \\
0 & a 1_{\mathfrak{m}}
\end{array}\right) \quad \text { and } \quad \widetilde{a 1_{R}}=\left(\begin{array}{cc}
a 1_{R} & 0 \\
0 & 1_{\mathfrak{m}}
\end{array}\right) ;
$$

see Example (2.7). In view of Definition (2.10) and Remark (2.11), the subgroup $\Xi$ of $\operatorname{Aut}_{R}(R \oplus \mathfrak{m})_{\text {ab }}$ is therefore generated by all elements of the form

$$
\xi_{a}:=\left(\widetilde{a 1_{\mathfrak{m}}}\right)\left(\widetilde{a 1_{R}}\right)^{-1}\left(\widetilde{a 1_{\mathfrak{m}}}\right)=\left(\begin{array}{cc}
a^{-1} 1_{R} & 0 \\
0 & a^{2} 1_{\mathfrak{m}}
\end{array}\right) \quad \text { where } \quad a \in k^{*} .
$$

Denote by $\omega$ the composite of the isomorphisms,

$$
\operatorname{Aut}_{R}(R \oplus \mathfrak{m})_{\mathrm{ab}} \stackrel{\delta}{\simeq} k^{*} \oplus \operatorname{Aut}_{R}(\mathfrak{m}) \underset{\cong}{\longrightarrow} \oplus k^{*},
$$

where $\delta$ is the isomorphism from Proposition (9.6). As $\omega\left(\xi_{a}\right)=\left(a^{-1}, a\right)$ we get that $\omega(\Xi)=\left\{\left(a^{-1}, a\right) \mid a \in k^{*}\right\}$ and thus $\omega$ induces the first group isomorphism below,

$$
\operatorname{Aut}_{R}(R \oplus \mathfrak{m})_{\mathrm{ab}} / \Xi \underset{\bar{\omega}}{\stackrel{\longrightarrow}{\cong}}\left(k^{*} \oplus k^{*}\right) / \omega(\Xi) \underset{\cong}{\stackrel{\chi}{\cong}} k^{*} ;
$$

the second isomorphism is induced by the surjective homomorphism $k^{*} \oplus k^{*} \rightarrow k^{*}$, given by $(b, a) \mapsto b a$, whose kernel is exactly $\omega(\Xi)$. In view of Theorem (2.12) and the isomorphisms $\bar{\omega}$ and $\chi$ above, it follows that $\mathrm{K}_{1}(\bmod R) \cong k^{*}$.

Theorem (2.12) asserts that $\mathrm{K}_{1}$ (inc) may be identified with the homomorphism

$$
\lambda: R^{*} \longrightarrow \operatorname{Aut}_{R}(R \oplus \mathfrak{m})_{\mathrm{ab}} / \Xi \quad \text { given by } \quad r \longmapsto\left(\begin{array}{cc}
r 1_{R} & 0 \\
0 & 1_{\mathfrak{m}}
\end{array}\right) .
$$

It remains to note that the isomorphism $\chi \bar{\omega}$ identifies $\lambda$ with the homomorphism $\mu$ described in the example, indeed, one has $\chi \bar{\omega} \lambda=\mu$.

Example (10.2) shows that for $R=k[X] /\left(X^{2}\right)$ the canonical homomorphism,

$$
R^{*} \cong \mathrm{K}_{1}(\operatorname{proj} R) \stackrel{\mathrm{K}_{1}(\mathrm{inc})}{\longrightarrow} \mathrm{K}_{1}(\bmod R) \cong k^{*},
$$

is not an isomorphism. It turns out that if $k$ is algebraically closed with characteristic zero, then there exists a non-canonical isomorphism between $R^{*}$ and $k^{*}$.

(10.3) Proposition. Let $R=k[X] /\left(X^{2}\right)$ where $k$ is an algebraically closed field with characteristic $p \geqslant 0$. The following assertions hold.

(a) If $p>0$, then the groups $R^{*}$ and $k^{*}$ are not isomorphic.

(b) If $p=0$, then there exists a (non-canonical) group isomorphism $R^{*} \cong k^{*}$. 
Proof. There is a group isomorphism $R^{*} \rightarrow k^{*} \oplus k^{+}$given by $a+b X \mapsto(a, b / a)$, where $k^{+}$denotes the underlying abelian group of the field $k$.

"(a)": Let $\varphi=\left(\varphi_{1}, \varphi_{2}\right): k^{*} \rightarrow k^{*} \oplus k^{+}$be any group homomorphism. As $k$ is algebraically closed, every element in $x \in k^{*}$ has the form $x=y^{p}$ for some $y \in k^{*}$. Therefore $\varphi(x)=\varphi\left(y^{p}\right)=\varphi(y)^{p}=\left(\varphi_{1}(y), \varphi_{2}(y)\right)^{p}=\left(\varphi_{1}(y)^{p}, p \varphi_{2}(y)\right)=\left(\varphi_{1}(x), 0\right)$, which shows that $\varphi$ is not surjective.

"(b)": Since $p=0$ the abelian group $k^{+}$is divisible and torsion free. Therefore $k^{+} \cong \mathbb{Q}^{(I)}$ for some index set $I$. There exist algebraic field extensions of $\mathbb{Q}$ of any finite degree, and these are all contained in the algebraically closed field $k$. Thus $|I|=\operatorname{dim}_{\mathbb{Q}} k$ must be infinite, and it follows that $|I|=|k|$.

The abelian group $k^{*}$ is also divisible, but it has torsion. Write $k^{*} \cong T \oplus\left(k^{*} / T\right)$, where $T=\left\{x \in k^{*} \mid \exists n \in \mathbb{N}: x^{n}=1\right\}$ is the torsion subgroup of $k^{*}$. For the divisible torsion free abelian group $k^{*} / T$ one has $k^{*} / T \cong \mathbb{Q}^{(J)}$ for some index set $J$. It is not hard to see that $|J|$ must be infinite, and hence $|J|=\left|k^{*} / T\right|$. As $|T|=\aleph_{0}$ it follows that $|k|=\left|k^{*}\right|=\aleph_{0}+|J|=|J|$.

Since $|J|=|k|=|I|$ one gets $k^{*} \cong T \oplus \mathbb{Q}^{(J)} \cong T \oplus \mathbb{Q}^{(J)} \oplus \mathbb{Q}^{(I)} \cong k^{*} \oplus k^{+}$.

The artinian ring $R=k[X] /\left(X^{2}\right)$ from Example (10.2) has length $\ell=2$ and this power is also involved in the description of the homomorphism $\mu=\mathrm{K}_{1}$ (inc). The next result shows that this is no coincidence. As Proposition (10.4) might be well-known to experts, and since we do not really need it, we do not give a proof.

(10.4) Proposition. Let $(R, \mathfrak{m}, k)$ be a commutative artinian local ring of length $\ell$. The group homomorphism $R^{*} \cong \mathrm{K}_{1}(\operatorname{proj} R) \rightarrow \mathrm{K}_{1}(\bmod R) \cong k^{*}$ induced by the inclusion inc: $\operatorname{proj} R \rightarrow \bmod R$ is the composition of the homomorphisms,

$$
R^{*} \stackrel{\pi}{\longrightarrow} k^{*} \stackrel{(\cdot)^{\ell}}{\longrightarrow} k^{*},
$$

where $\pi: R \rightarrow R / \mathfrak{m}=k$ is the canonical quotient map and $(\cdot)^{\ell}$ is the $\ell^{\prime}$ th power.

Our next example is a non-artinian ring, namely the simple curve singularity of type $\left(A_{2}\right)$ studied by e.g. Herzog [19, Satz 1.6] and Yoshino [32, prop. (5.11)].

(10.5) Example. Let $R=k \llbracket T^{2}, T^{3} \rrbracket$ where $k$ is an algebraically closed field with $\operatorname{char}(k) \neq 2$. Denote by inc: $\operatorname{proj} R \rightarrow \bmod R$ the inclusion functor. The homomorphism $\mathrm{K}_{1}$ (inc) may be identified with the inclusion map,

$$
\mu: R^{*}=k \llbracket T^{2}, T^{3} \rrbracket^{*} \hookrightarrow k \llbracket T \rrbracket^{*} .
$$

Proof. The maximal ideal $\mathfrak{m}=\left(T^{2}, T^{3}\right)$ is the only non-free indecomposable maximal Cohen-Macaulay $R$-module, so $M=R \oplus \mathfrak{m}$ is a representation generator for MCM $R$; see (2.1.1). Even though $T$ is not an element in $R=k \llbracket T^{2}, T^{3} \rrbracket$, multiplication by $T$ is a well-defined endomorphism of $\mathfrak{m}$. Thus there is a ring homomorphism,

$$
\chi: k \llbracket T \rrbracket \longrightarrow \operatorname{End}_{R}(\mathfrak{m}) \quad \text { given by } \quad h \longmapsto h 1_{\mathfrak{m}} .
$$

It is not hard to see that $\chi$ is injective. To prove that it is surjective, i.e. that one has $\operatorname{End}_{R}(\mathfrak{m}) / k \llbracket T \rrbracket=0$, note that there is a short exact sequence of $R$-modules,

$$
0 \longrightarrow k \llbracket T \rrbracket / R \longrightarrow \operatorname{End}_{R}(\mathfrak{m}) / R \longrightarrow \operatorname{End}_{R}(\mathfrak{m}) / k \llbracket T \rrbracket \longrightarrow 0 .
$$

To see that $\operatorname{End}_{R}(\mathfrak{m}) / k \llbracket T \rrbracket=0$, it suffices to argue that the $R$-module $\operatorname{End}_{R}(\mathfrak{m}) / R$ is simple. As noted in the beginning of the proof of Proposition (9.6), the inclusion $\mathfrak{m} \hookrightarrow R$ induces an isomorphism $\operatorname{End}_{R}(\mathfrak{m}) \cong \operatorname{Hom}_{R}(\mathfrak{m}, R)$, so by applying 
$\operatorname{Hom}_{R}(-, R)$ to the short exact sequence $0 \rightarrow \mathfrak{m} \rightarrow R \rightarrow k \rightarrow 0$, it follows that

$$
\operatorname{End}_{R}(\mathfrak{m}) / R \cong \operatorname{Ext}_{R}^{1}(k, R) .
$$

The latter module is isomorphic to $k$ since $R$ is a 1-dimensional Gorenstein ring.

Note that via the isomorphism $\chi$, the group $k \llbracket T \rrbracket^{*}$ corresponds to $\operatorname{Aut}_{R}(\mathfrak{m})$.

The Auslander-Reiten sequence ending in $\mathfrak{m}$ is

$$
0 \longrightarrow \mathfrak{m} \stackrel{(1-T)^{t}}{\longrightarrow} R \oplus \mathfrak{m} \stackrel{\left(T^{2} T\right)}{\longrightarrow} \mathfrak{m} \longrightarrow 0 .
$$

Since the Auslander-Reiten homomorphism $\Upsilon=\left(\begin{array}{c}-1 \\ 1\end{array}\right): \mathbb{Z} \rightarrow \mathbb{Z}^{2}$ is injective, Theorem (2.12) can be applied. We regard elements in $R \oplus \mathfrak{m}$ as column vectors. Let $\alpha=h 1_{\mathfrak{m}} \in \operatorname{Aut}_{R}(\mathfrak{m})$, where $h \in k \llbracket T \rrbracket^{*}$, be given. Write $h=f+g T$ for some $f \in R^{*}$ and $g \in R$. It is straightforward to verify that there is a commutative diagram,

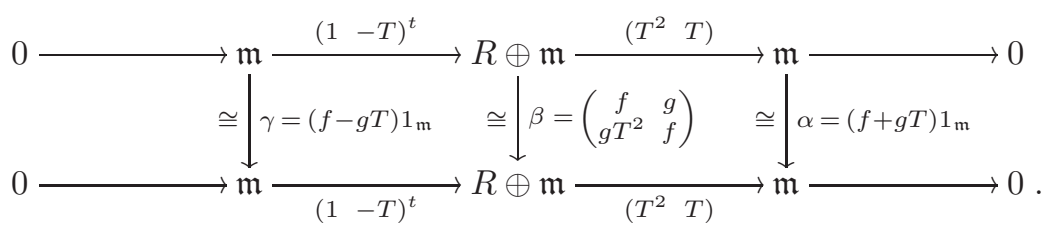

Note that $\beta$ really is an automorphism; indeed, its inverse is given by

$$
\beta^{-1}=\left(f^{2}-g^{2} T^{2}\right)^{-1}\left(\begin{array}{cc}
f & -g \\
-g T^{2} & f
\end{array}\right) .
$$

We now apply the tilde construction (2.6) to $\alpha, \beta$, and $\gamma$; by Example (2.7) we get:

$$
\tilde{\alpha}=\left(\begin{array}{cc}
1 & 0 \\
0 & f+g T
\end{array}\right), \quad \tilde{\beta}=\beta, \quad \text { and } \quad \tilde{\gamma}=\left(\begin{array}{cc}
1 & 0 \\
0 & f-g T
\end{array}\right) .
$$

In view of Definition (2.10) and Remark (2.11), the subgroup $\Xi$ of $\operatorname{Aut}_{R}(R \oplus \mathfrak{m})_{\mathrm{ab}}$ is therefore generated by all the elements

$$
\xi_{h}:=\tilde{\alpha} \tilde{\beta}^{-1} \tilde{\gamma}=\left(f^{2}-g^{2} T^{2}\right)^{-1}\left(\begin{array}{cc}
f & -g(f-g T) \\
-g T^{2}(f+g T) & f\left(f^{2}-g^{2} T^{2}\right)
\end{array}\right) .
$$

Denote by $\omega$ the composite of the isomorphisms,

$$
\operatorname{Aut}_{R}(R \oplus \mathfrak{m})_{\mathrm{ab}} \underset{\cong}{\stackrel{\delta}{\cong}} k^{*} \oplus \operatorname{Aut}_{R}(\mathfrak{m}) \stackrel{1 \oplus \chi^{-1}}{\cong} k^{*} \oplus k \llbracket T \rrbracket^{*}
$$

where $\delta$ is the isomorphism from Proposition (9.6). Note that $\delta\left(\xi_{h}\right)=\left([f]_{\mathfrak{m}}, 1_{\mathfrak{m}}\right)=$ $\left(h(0), 1_{\mathfrak{m}}\right)$ and hence $\omega\left(\xi_{h}\right)=(h(0), 1)$. It follows that $\omega(\Xi)=k^{*} \oplus\{1\}$ and thus $\omega$ induces a group isomorphism,

$$
\bar{\omega}: \operatorname{Aut}_{R}(R \oplus \mathfrak{m})_{\mathrm{ab}} / \Xi \stackrel{\cong}{\longrightarrow}\left(k^{*} \oplus k \llbracket T \rrbracket^{*}\right) / \omega(\Xi)=k \llbracket T \rrbracket^{*} .
$$

In view of this isomorphism, Theorem (2.12) shows that $\mathrm{K}_{1}(\bmod R) \cong k \llbracket T \rrbracket^{*}$. Theorem (2.12) also asserts that $\mathrm{K}_{1}$ (inc) may be identified with the homomorphism

$$
\lambda: R^{*} \longrightarrow \operatorname{Aut}_{R}(R \oplus \mathfrak{m})_{\mathrm{ab}} / \Xi \quad \text { given by } \quad f \longmapsto\left(\begin{array}{cc}
f 1_{R} & 0 \\
0 & 1_{\mathfrak{m}}
\end{array}\right) .
$$

It remains to note that the isomorphism $\bar{\omega}$ identifies $\lambda$ with the inclusion map $\mu$ described in the example, indeed, one has $\bar{\omega} \lambda=\mu$. 


\section{ACKNOWLEDGEMENTS}

It is a pleasure to thank Peter Jørgensen without whom this paper could not have been written. However, Peter did not wish to be a coauthor of this manuscript. We are also grateful to Marcel Bökstedt and Charles A. Weibel for valuable input on the Gersten-Sherman transformation, and to Christian U. Jensen for pointing out Proposition (10.3). Finally, we thank Viraj Navkal and the anonymous referee for their thoughtful comments and for making us aware of the paper 23.

\section{REFERENCES}

[1] Maurice Auslander, Representation theory of Artin algebras. I, II, Comm. Algebra 1 (1974), 177-268; ibid. 1 (1974), 269-310.

$[2]$, Rational singularities and almost split sequences, Trans. Amer. Math. Soc. 293 (1986), no. 2, 511-531.

[3] Maurice Auslander and Ragnar-Olaf Buchweitz, The homological theory of maximal CohenMacaulay approximations, Mém. Soc. Math. France (N.S.) (1989), no. 38, 5-37, Colloque en l'honneur de Pierre Samuel (Orsay, 1987).

[4] Maurice Auslander and Idun Reiten, Grothendieck groups of algebras and orders, J. Pure Appl. Algebra 39 (1986), no. 1-2, 1-51.

[5] _ Almost split sequences for rational double points, Trans. Amer. Math. Soc. 302 (1987), no. 1, 87-97.

[6] Maurice Auslander, Idun Reiten, and Sverre O. Smalø, Representation theory of Artin algebras, Cambridge Studies in Advanced Mathematics, vol. 36, Cambridge University Press, Cambridge, 1995.

[7] Hyman Bass, Algebraic K-theory, W. A. Benjamin, Inc., New York-Amsterdam, 1968.

[8] David J. Benson, Representations and cohomology. I, second ed., Cambridge Studies in Advanced Mathematics, vol. 30, Cambridge University Press, Cambridge, 1998, Basic representation theory of finite groups and associative algebras.

[9] Michael C. R. Butler, Grothendieck groups and almost split sequences, Integral representations and applications (Oberwolfach, 1980), Lecture Notes in Math., vol. 882, Springer, Berlin, 1981, pp. 357-368.

[10] Nuri Cimen, One-dimensional rings of finite Cohen-Macaulay type, ProQuest LLC, Ann Arbor, MI, 1994, Thesis (Ph.D.)-The University of Nebraska - Lincoln.

[11] _ One-dimensional rings of finite Cohen-Macaulay type, J. Pure Appl. Algebra 132 (1998), no. 3, 275-308.

[12] Ju. A. Drozd and Andrei V. Roĭter, Commutative rings with a finite number of indecomposable integral representations, Izv. Akad. Nauk SSSR Ser. Mat. 31 (1967), 783-798.

[13] Edgar E. Enochs and Overtoun M. G. Jenda, Relative homological algebra, de Gruyter Expositions in Mathematics, vol. 30, Walter de Gruyter \& Co., Berlin, 2000.

[14] Hélène Esnault, Reflexive modules on quotient surface singularities, J. Reine Angew. Math. 362 (1985), 63-71.

[15] William Fulton and Joe Harris, Representation theory, Graduate Texts in Mathematics, vol. 129, Springer-Verlag, New York, 1991, A first course, Readings in Mathematics.

[16] Pierre Gabriel, Des catégories abéliennes, Bull. Soc. Math. France 90 (1962), 323-448.

[17] Stephen M. Gersten, Higher $K$-theory of rings, Algebraic $K$-theory, I: Higher $K$-theories (Proc. Conf. Seattle Res. Center, Battelle Memorial Inst., 1972), Springer, Berlin, 1973, pp. 3-42. Lecture Notes in Math., Vol. 341.

[18] Edward L. Green and Irving Reiner, Integral representations and diagrams, Michigan Math. J. 25 (1978), no. 1, 53-84.

[19] Jürgen Herzog, Ringe mit nur endlich vielen Isomorphieklassen von maximalen, unzerlegbaren Cohen-Macaulay-Moduln, Math. Ann. 233 (1978), no. 1, 21-34.

[20] Tsit-Yuen Lam, A first course in noncommutative rings, second ed., Graduate Texts in Mathematics, vol. 131, Springer-Verlag, New York, 2001.

[21] Graham J. Leuschke, Endomorphism rings of finite global dimension, Canad. J. Math. 59 (2007), no. 2, 332-342. 
[22] Oscar Litoff, On the commutator subgroup of the general linear group, Proc. Amer. Math. Soc. 6 (1955), 465-470.

[23] Viraj Navkal, K'-theory of a local ring of finite Cohen-Macaulay type, preprint (2012) arXiv:1108.2000v2 [math.KT] (see http://www.math.ucla.edu/ iraj/ for the latest version).

[24] Daniel Quillen, Higher algebraic K-theory. I, Algebraic K-theory, I: Higher $K$-theories (Proc. Conf., Battelle Memorial Inst., Seattle, Wash., 1972), Springer, Berlin, 1973, pp. 85-147. Lecture Notes in Math., Vol. 341.

[25] Jonathan Rosenberg, Algebraic K-theory and its applications, Graduate Texts in Mathematics, vol. 147, Springer-Verlag, New York, 1994.

[26] Clayton Sherman, Group representations and algebraic K-theory, Algebraic $K$-theory, Part I (Oberwolfach, 1980), Lecture Notes in Math., vol. 966, Springer, Berlin, 1982, pp. 208-243.

[27] Vasudevan Srinivas, Algebraic K-theory, second ed., Progress in Mathematics, vol. 90, Birkhäuser Boston Inc., Boston, MA, 1996.

[28] Leonid N. Vaserstein, On the stabilization of the general linear group over a ring, Math. USSR-Sb. 8 (1969), 383-400.

[29] _ On the Whitehead determinant for semi-local rings, J. Algebra 283 (2005), no. 2, 690-699.

[30] Roger Wiegand, Noetherian rings of bounded representation type, Commutative algebra (Berkeley, CA, 1987), Math. Sci. Res. Inst. Publ., vol. 15, Springer, New York, 1989, pp. 497516.

[31] _ One-dimensional local rings with finite Cohen-Macaulay type, Algebraic geometry and its applications (West Lafayette, IN, 1990), Springer, New York, 1994, pp. 381-389.

[32] Yuji Yoshino, Cohen-Macaulay modules over Cohen-Macaulay rings, London Mathematical Society Lecture Note Series, vol. 146, Cambridge University Press, Cambridge, 1990.

Department of Mathematical Sciences, Faculty of Science, University of Copenhagen, Universitetsparken 5, 2100 Copenhagen $\varnothing$, Denmark

E-mail address: holm@math.ku.dk

$U R L:$ http://www.math.ku.dk/ holm/ 